\title{
Dissertation
}

\section{Stoßwellenuntersuchungen und Modellierung der Pyrolyse von Pentafluorethan und 2-H-Heptafluorpropan}

\author{
zur Erlangung des \\ mathematisch-naturwissenschaftlichen Doktorgrades \\ Doctor rerum naturalium \\ der \\ Georg-August-Universität Göttingen \\ im \\ Promotionsprogramm Chemie \\ der Georg-August University School of Science (GAUSS)
}

vorgelegt von

Elsa Tellbach (geb. Rusch)

aus Greifswald

angefertigt im

Institut für Physikalische Chemie

der Georg-August-Universität zu Göttingen

2013 


\section{Betreuungsausschuss}

Prof. J. Troe, Abt. Troe, Institut für Physikalische Chemie

(Name, Abteilung/Arbeitsgruppe, Institution)

Prof. J. Schröder, Abt. Troe, Institut für Physikalische Chemie

(Name, Abteilung/Arbeitsgruppe, Institution)

\section{Mitglieder der Prüfungskommission}

Referent/in: Prof. J. Troe, Abt. Troe, Institut für Physikalische Chemie (Namen, Abteilung/Arbeitsgruppe, Institution)

Korreferent/in: Prof. J. Schröder, Abt. Troe, Institut für Physikalische Chemie (Name, Abteilung/Arbeitsgruppe, Institution)

\section{Weitere Mitglieder der Prüfungskommission}

Prof. A. Wodtke, Abt. Wodtke, Institut für Physikalische Chemie

(Name, Abteilung/Arbeitsgruppe, Institution)

Prof. D. Schwarzer, Abt. Wodtke, Institut für Physikalische Chemie (Name, Abteilung/Arbeitsgruppe, Institution)

Prof. P. Botschwina, Abt. Botschwina, Institut für Physikalische Chemie (Name, Abteilung/Arbeitsgruppe, Institution)

Prof. B. Abel, Abt. Abel, Leibniz Instiut, Leipzig (Name, Abteilung/Arbeitsgruppe, Institution)

Tag der mündlichen Prüfung: 13.12.2013 


\section{Danksagung}

Herrn Prof. Dr. J. Troe möchte ich für die Aufnahme in seine Arbeitsgruppe, die hervorragenden experimentellen Bedingungen, die stete Unterstützung in allen Umständen und die freundliche Arbeitsatmosphäre in unserer kleinen Gruppe danken. Ohne die zahllosen Geschichten und Anekdoten wäre es wohl nur halb so schön gewesen.

Herrn Carlos Cobos danke ich ganz besonders für seine Arbeit bezüglich der hier verwendeten quantenchemischen Rechnungen, die einen Großteil der Erklärungen lieferte.

Prof. Dr. J. Schröder danke ich für die freundliche Betreuung, Hilfsbereitschaft, Unterstützung und netten Gespräche zwischendurch.

Prof. Dr. K. Luther danke ich für die erfrischenden Gespräche über die kleinen und großen Geheimnisse der Stoßwellenapparatur.

Meinem lieben Kollegen Lars gilt ein ganz besonderer Dank für die immerwährende Hilfe und Unterstützung zu jeder Tages- und Nachtzeit, für lange und unterhaltsame Messabende, ausgleichende Lauftrainings, unermüdliche Erklärungen, seine bedingungslose Freundschaft und für all den Mut, den er mir in zweifelnden Momenten zugesprochen hat.

Allen Kollegen danke ich für ihre Herzlichkeit, die eine freundliche Atmosphäre geschaffen hat.

Herrn R. Bürsing möchte ich für die technische Unterstützung und die vielen kleinen Hilfestellungen danken.

Mein Dank geht auch an die gewissenhaft arbeitenden Werkstätten in diesem Haus.

Danken möchte ich besonders Milo für seine tatkräftige Hilfe.

Ich danke meinem Mann für die aufopferungsvolle Unterstützung, in dem er mir immer den Rücken freigehalten hat, und hinter mir stand, wann immer ich es brauchte. Ich danke Dir für all die durchwachten Nächte, während ich schlafen konnte und für warmes Abendbrot, wann immer ich nach Hause kam - egal zu welcher Uhrzeit.

Ich danke meiner Familie, die immer an mich geglaubt hat - und für Acht-UhrHeißgetränke. 



\section{Zusammenfassung}

Die vorliegende Arbeit beschäftigt sich mit dem thermischen Zerfall von Fluorkohlenstoffverbindungen. Die Pyrolyse wird in einem Stoßrohr bei hohen Temperaturen und verschiedenen Drücken mittels zeitaufgelöster UV-Absorptionsspektroskopie von $\mathrm{CF}_{2}$ untersucht.

Nach einer Einführung in die verwendeten experimentellen Techniken, beschäftigt sich der erste Abschnitt mit Pentafluorethan, welches nach der Gleichung:

$$
\mathrm{C}_{2} \mathrm{~F}_{5} \mathrm{H} \longrightarrow 2 \mathrm{CF}_{2}+\mathrm{H}+\mathrm{F}
$$

zerfällt. Dabei kann als Geschwindigkeitskonstante für den ersten Reaktionsschritt

$$
\mathrm{CF}_{3} \mathrm{CF}_{2} \mathrm{H} \longrightarrow \mathrm{CF}_{3}+\mathrm{CF}_{2} \mathrm{H}
$$

ein Wert von

$$
k=2.2 \cdot 10^{18} \cdot \exp \left(-410 \mathrm{~kJ} \mathrm{~mol}{ }^{-1} / R T\right) \mathrm{s}^{-1}
$$

ermittelt werden. Bei höheren Reaktandenkonzentrationen folgt eine Kettenreaktion, deren wahrscheinlicher Mechanismus mit Hilfe von quantenchemischen Berechnungen modelliert wurde. Die Untersuchungen erstrecken sich über einen Druck- und Temperaturbereich von $[\mathrm{Ar}]=2 \cdot 10^{-5}-10 \cdot 10^{-5} \mathrm{~mol} / \mathrm{cm}^{3}$ (entspricht $3-12$ bar)bei $1200-2000 \mathrm{~K}$.

Der zweite Abschnitt befasst sich mit 2H-Heptafluorpropan, welches in einem ähnlichen Druck- und Temperaturbereich untersucht wird. $\mathrm{C}_{3} \mathrm{HF}_{7}$ zerfällt ebenfalls zu $\mathrm{CF}_{2}$ entsprechend der Gleichung

$$
\mathrm{C}_{3} \mathrm{~F}_{7} \mathrm{H} \longrightarrow 3 \mathrm{CF}_{2}+\mathrm{H}+\mathrm{F}
$$


Der erste Reaktionsschritt ist auch hierbei ein C-C-Bindungsbruch

$$
\mathrm{CF}_{3} \mathrm{CHFCF}_{3} \longrightarrow \mathrm{CF}_{3} \mathrm{CFH}+\mathrm{CF}_{3}
$$

mit einer Geschwindigkeitskonstante von

$$
k=10^{15.7} \cdot \exp (-42800 \mathrm{~K} / T) \mathrm{s}^{-1}
$$

Der gesamte Mechanismus der Pyrolyse des $\mathrm{C}_{3} \mathrm{HF}_{7}$ verläuft jedoch komplexer, wobei erneut Anzeichen für einen Kettenmechanismus gefunden wurden. 


\section{Inhaltsverzeichnis}

1 Einleitung 1

1.1 Fluorkohlenwasserstoffe als Kältemittel . . . . . . . . . . . 1

1.1.1 Nomenklatur . . . . . . . . . . . . . . . 2

1.1.2 Aktuelle Forschung . . . . . . . . . . . .

1.2 Fluorkohlenwasserstoffe als Löschmittel . . . . . . . . . B

1.3 Einfluss auf den Treibhauseffekt . . . . . . . . . . 4

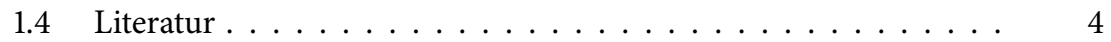

2 Experimentelle Technik 7

2.1 Die Stoßwellentechnik . . . . . . . . . . . . . . . 7

2.1.1 Berechnung der Zustandsdaten $\ldots \ldots \ldots \ldots \ldots$

2.1.2 Experimenteller Aufbau . . . . . . . . . . . . . 11

2.1.2.1 Signalverarbeitung . . . . . . . . . . . 14

2.1.2.2 Verwendete Chemikalien ........... 16

3 Auswertung 17

3.1 Allgemeines . . . . . . . . . . . . . . . 17

3.1.1 Experimentelle Bedingungen . . . . . . . . . . 17

3.1.1.1 Der Absorptionskoeffizient . . . . . . . . . 17

3.1 .2 Signalauswertung . . . . . . . . . . . . 18

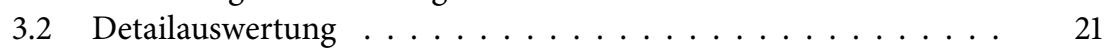

3.2.1 Bestimmung der Ausbeute . . . . . . . . . . . 21

3.2.2 Visuelle Bewertung der Messdaten . . . . . . . . . 2 24

3.2.3 Bestimmung der Geschwindigkeitskonstanten . . . . . 25

4 Diskussion 29

4.1 Resultate zur Pyrolyse des Pentafluorethans . . . . . . . . . 29

4.1.1 Modellierung der primären Reaktionsschritte . . . . . . 32

4.1.2 Modellierung des Sekundärmechanismus . . . . . . . . 37

4.2 Resultate zur Pyrolyse des Heptafluorethans . . . . . . . . . 40

4.2.1 Modellierung der primären Reaktionsschritte . . . . . . 43

4.2.2 Modellierung des Sekundärmechanismus . . . . . . . 46

5 Ausblick 49 
A Molekulare Parameter für die Modellierung 53

A.1 Harmonische Schwingungsfrequenzen . . . . . . . . . 53

A.2 Rotationskonstanten . . . . . . . . . . . . . . 54

Abbildungsverzeichnis 61

Tabellenverzeichnis 63 


\section{Kapitel 1}

\section{Einleitung}

Im Rahmen dieser Arbeit werden zwei teilfluorierte Kohlenwasserstoffe auf ihre Reaktionsgeschwindigkeit und den zugrunde liegenden Mechanismus untersucht. Sowohl $\mathrm{C}_{2} \mathrm{HF}_{5}$, als auch $\mathrm{C}_{3} \mathrm{HF}_{7}$, sind wegen ihrer möglichen breiten Anwendungsbereiche interessant. Sie sind nicht nur als Kältemittel in der Diskussion, sondern auch als Ersatzstoffe für Halone in der Brandbekämpfung. Allerdings besitzen fluorierte Kohlenwasserstoffe (FKW) einen nicht zu unterschätzenden Einfluss auf die Atmosphäre und den Treibhauseffekt. Es ist daher wichtig und notwendig, diese Stoffe und ihr Reaktionsverhalten bei unterschiedlichsten Bedingungen zu untersuchen und zu verstehen. Um einen Überblick über die verschiedenen Anwendungsgebiete und eventuelle Umweltproblematiken zu bekommen, wird im Folgenden kurz die Verwendung von FKWs als Kälte- und Feuerlöschmittel, sowie auf ihren Einfluss auf die Atmosphäre eingegangen.

\subsection{Fluorkohlenwasserstoffe als Kältemittel}

Kältemittel sind ubiquitär vorhanden und werden in vielen industriellen Anwendungen eingesetzt, sowohl im heimischen Kühlschrank, der Klimaanlage oder auch in großmaßstäblichen Kühlräumen. Ein genaues Verständnis ihrer Wirkung und der Auswirkungen auf das atmosphärische Gleichgewicht ist daher unabdingbar.

Kältemittel sind nach DIN EN 378-1 Abs. 3.7.1 definiert als: „Fluid, das zur Wärmeübertragung in einer Kälteanlage eingesetzt wird und das bei niedriger Temperatur und niedrigem Druck Wärme aufnimmt und bei höherer Temperatur und höherem Druck Wärme abgibt, wobei üblicherweise Zustandsänderungen des Fluids erfolgen." DIN 8960 Abs. 3.1 bezeichnet Kältemittel auch als: „Arbeitsmedium, das in einem Kältemaschinenprozess bei niedriger Temperatur und niedrigem Druck Wärme aufnimmt und bei höherer Temperatur und höherem Druck Wärme abgibt." Beide DIN-Normen beziehen sich speziell auf Kompressions-Kältemaschinen. 
Es gibt verschiedene physikalisch-chemische Reaktionen, die in Kälteanlangen genutzt werden. Bei reinen Kältemitteln wird Wärme durch Verdampfung bei niedrigem Druck und Temperatur aufgenommen. Das Kältemittel regeneriert sich durch Verflüssigung in einer Verdichter-Verflüssiger-Reihe. Beim Einsatz einer Kältemischung erfolgt die Reaktion chemisch durch Mischungs- oder Lösungsreaktion. Die Regeneration erfolgt dann durch Entmischung in einem thermischen Verdichter.

Weit verbreitet sind Kompressionskälteanlagen. Der Kältemitteldampf wird hierbei in einem Kreisprozess durch die Anlage geführt. Zuerst gelangt das Kühlmittel durch einen Verdichter. Das so komprimierte Gas wird anschließend im Verflüssiger kondensiert und danach über eine Drossel expandiert, wobei es abkühlt und verdampft. Im zweiten Wärmeüberträger nimmt das nun kalte Kühlmittel Wärme aus dem Kühlraum auf und der Prozess beginnt von neuem. Nach dem zweiten Hauptsatz der Thermodynamik muss für diesen Prozess Energie von außen (mechanische Arbeit) zugeführt werden.

\subsubsection{Nomenklatur}

Kältemittel folgen einer eigenen Nomenklatur, welche in DIN 8960 Abs. 6 festgelegt ist. Um Rückschlüsse auf die verwendeten Chemikalien zu ziehen, ist es nötig, sich die folgenden Regeln zu vergegenwärtigen. Die allgemeine Bezeichnung eines Kältemittels lautet „R-zzzbb“ - wobei „R“ für refrigerant (engl. Kältemittel) und „, $\mathrm{Z}$ “ für drei (in Sonderfällen zwei, vier) Ziffern steht. „bb“ kennzeichnet Isomere gleicher Summenformel.

Als Beispiel zur Nomenklatur dient das in dieser Arbeit verwendete Pentafluorethan mit der Kältemittelbezeichnung R-125. Fluorkohlenwasserstoffe als Kältemittel werden generell nach folgender Formel benannt:

$$
R-(C-1)(H+1)(F)
$$

wobei C, H und F für Kohlenstoff-, Wasserstoff- und Fluoratome stehen.

R-125 besteht demnach aus zwei Kohlenstoffatomen, einem Wasserstoffatom und fünf Fluoratomen.

Zur Benennung von cyclischen, ungesättigten oder bromhaltigen Verbindungen sowie anorganischen Kältemitteln existieren eine Reihe von erweiterten Nomenklaturregeln, auf die hier nicht weiter eingegangen werden soll. 


\subsubsection{Aktuelle Forschung}

In der Richtlinie 2006/40/EG des Europäischen Parlaments vom 17. Mai 2006 wird angeordnet, dass in PKW-Klimaanlagen nur noch Kältemittel mit einem GWP (globalwarming potential) unterhalb von 150 genutzt werden dürfen. Damit muss mittelfristig das bisher verwendete R-134a (Tetrafluorethan, GWP=1300) ersetzt werden. Als neues Kältemittel in PKW-Klimaanlagen ist R-1234yf (Tetrafluorpropen) bzw. R-744 $\left(\mathrm{CO}_{2}\right)$ vorgesehen. Aufgrund der Brennbarkeit von R-1234yf gibt es eine anhaltende Diskussion einzelner Automobilhersteller über die Verwendung dieses Kältemittels. R-744 kann zum heutigen Zeitpunkt noch nicht als ein vollwertiger Ersatz gelten, da keine serienfertigen PKW-Klimaanlagen existieren, die den erhöhten Druckanforderungen von $\mathrm{CO}_{2}$ entsprechen.

Nachdem in den 1980er Jahren bekannt wurde, dass Fluorchlorkohlenwasserstoffe (FCKW) ein hohes Ozonabbaupotential (ODP - ozone depletion potential) besitzen und darüber hinaus den Treibhauseffekt verstärken, wurde in der FCKW-HalonVerbots-Verordnung (seit 1. Dezember 2006 die Chemikalien-Ozonschichtverordnung) geregelt, dass in Deutschland keine vollhalogenierten Fluorchlorkohlenwasserstoffe mehr als Kältemittel Verwendung finden dürfen.

Deshalb wird verstärkt an teilfluorierten Kohlenwasserstoffen und ihrer Eignung als Kältemittel geforscht. Sie besitzen kein Ozonabbaupotential, zeigen allerdings einen größeren Einfluss auf den Treibhauseffekt. Es ist daher unumgänglich, weiter an einer Lösung zu arbeiten, mit der Zielsetzung, Kältemittel zu finden, die klimaverträglich sind und möglichst wenig gefährliche Eigenschaften (wie Brennbarkeit oder Explosivität) zeigen.

\subsection{Fluorkohlenwasserstoffe als Löschmittel}

Nachdem 1989 das Montreal-Protokoll in Kraft trat, wurden die bromhaltigen Halone $\mathrm{CBrClF}_{2}$ und $\mathrm{CBrF}_{3}$ aufgrund ihres hohen Ozonabbaupotentials vom Markt genommen. Seither werden neue Halone auf ihren Einsatz als Feuerlöschmittel geprüft und inzwischen auch von der zuständigen EU-Kommission zugelassen. Darunter fällt auch das hier untersuchte $\mathrm{C}_{2} \mathrm{HF}_{5}$ [1-5].

Halone wirken als chemische Feuerlöschmittel, in dem sie eine Störung des Verbrennungsablaufs verursachen, z.B. in dem eine Kettenabbruchreaktion initiiert wird. Man unterscheidet dabei zwischen homogener und inhomogener Inhibition. Alle Halone unterdrücken Feuer durch homogene Inhibition, bei welcher als Kettenabbruch eine Rekombination der vorhandenen Radikale stattfindet. Der Vorteil dieses Verfahrens liegt im bedeutend kleineren Löschmittelvolumen, z. B. im Vergleich mit dem 
Ersticken durch $\mathrm{CO}_{2}$. Nachteilig hingegen ist die teilweise starke Wirkung der Halone auf den Treibhauseffekt (siehe auch 1.3). Auch besteht die Möglichkeit, dass sich das Halon bei langen Vorbrennzeiten des Feuers zersetzt und giftige Folgeprodukte entstehen [6].

\subsection{Einfluss auf den Treibhauseffekt}

Alle Kältemittel (und die bisher zugelassenen Halone mit Ausnahme von Novec 1230 (Perfluoro(2-methyl-3-pentanon))), außer $\mathrm{CO}_{2}$, haben ein GWP größer als 1, d.h. sie haben ein (im Vergleich zu $\mathrm{CO}_{2}$ ) erhöhtes Potential die globale Erwärmung zu verstärken. Dabei darf nicht übersehen werden, dass der Einfluss auf das Klima u.a. von der Verweilzeit der Chemikalie in der Atmosphäre abhängt. Das IPCC (Intergovernmental Panel on Climate Change) gibt daher GWP-Werte für Zeithorizonte von 20-100 Jahren an. Je nach Verweildauer in der Atmosphäre haben die Treibhausgase daher eher einen Einfluss auf das kurzfristige Klima oder die langfristige klimatische Entwicklung und Erwärmung der Erde. Ein Faktor zur Bestimmung von GWP-Werten ist das Absorptionsverhalten der Moleküle. Interessant ist hier besonders das atmosphärische Fenster von etwa $8-13 \mu \mathrm{mWellenlänge.} \mathrm{Gase,} \mathrm{die} \mathrm{die}$ von der Erdoberfläche reflektierte Wärmestrahlung in diesem Bereich absorbieren, tragen zur Erwärmung der Atmosphäre bei. Das in dieser Arbeit untersuchte R-125 $\left(\mathrm{C}_{2} \mathrm{HF}_{5}\right)$ hat ein GWP von 3500 bei einem Zeithorizont von 100 Jahren und einer Lebensdauer in der Atmosphäre von 36 Jahren [7].

In der vorliegenden Arbeit werden teilfluorierte Kohlenwasserstoffe untersucht. Nach der üblichen Definition gelten sie nicht als natürliche Kältemittel (im Gegensatz zu $\mathrm{NH}_{3}, \mathrm{CO}_{2}$ und $\mathrm{H}_{2} \mathrm{O}$ ), obwohl sie in der Natur von Mikroorganismen, Pflanzen und durch vulkanische Tätigkeit produziert und in die Atmosphäre entlassen werden [8|.

Ein umfassendes Verständnis der Reaktionsmechanismen, -zeiten und Lebensdauern von etwaigen Reaktionsprodukten ist unumgänglich für die korrekte Bewertung des Eintrags von teilfluorierten Kohlenwasserstoffen in die Atmosphäre.

\subsection{Literatur}

In dieser Arbeit liegt der Schwerpunkt des Interesses auf den primären Dissoziationsschritten von $\mathrm{C}_{2} \mathrm{HF}_{5}$ und $\mathrm{C}_{3} \mathrm{HF}_{7}$ bei Verbrennungsreaktionen, d.h. bei hohen Temperaturen von etwa 1200 - $2000 \mathrm{~K}$. Frühere experimentelle Arbeiten zur Pyrolyse von $\mathrm{C}_{2} \mathrm{HF}_{5}$ sind rar. Tschuikow-Roux [9, 10] untersuchte die Dissoziation mittels Single-Puls-Stoßwellen im Bereich von 1180 - $1470 \mathrm{~K}$ und Inomata et. al [11] 
beobachteten die Reaktion im Bereich von 1273 - 1373 K bei Atmosphärendruck in einer turbulenten Strömung. Ein Experiment, welches mit einer Gasströmung innerhalb eines UV-Photoelektronen-Spektrometers [5, 12| bei sehr kleinen Drücken von etwa $10^{-4}$ bis $10^{-3}$ Torr arbeitete, erlaubte eine Analyse der Reaktionsprodukte unter Niederdruckbedingungen. Dabei fand die Druckabhängigkeit der Reaktion allerdings keine Berücksichtigung. Die gemessenen Geschwindigkeitskonstanten aus [11] und [9] unterscheiden sich bis zu einem Faktor von fünf, so dass eine erneute Betrachtung der Reaktion sinnvoll erscheint. Als primärer Reaktionsschritt wurde bisher angenommen, dass HF eliminiert wird (1.1) und als zweiter Reaktionsweg bei hohen Temperaturen ein C-C-Bindungsbruch möglich ist (1.2),

$$
\begin{aligned}
& \mathrm{C}_{2} \mathrm{~F}_{5} \mathrm{H}+(\mathrm{M}) \longrightarrow \mathrm{C}_{2} \mathrm{~F}_{4}+\mathrm{HF}+(\mathrm{M}) \\
& \mathrm{C}_{2} \mathrm{~F}_{5} \mathrm{H}+(\mathrm{M}) \longrightarrow \mathrm{CF}_{2} \mathrm{H}+\mathrm{CF}_{3}+(\mathrm{M})
\end{aligned}
$$

In der vorliegenden Arbeit wird mittels UV-Spektroskopie die Entstehung von $\mathrm{CF}_{2}$ beobachtet [13], so dass die Möglichkeit besteht, den primären Reaktionsschritt anhand der schnellen Folgereaktion

$$
\mathrm{C}_{2} \mathrm{~F}_{4}+(\mathrm{M}) \longrightarrow 2 \mathrm{CF}_{2}+(\mathrm{M})
$$

direkt zu beobachten.

Die Datenlage für $\mathrm{C}_{3} \mathrm{HF}_{7}$ ist ähnlich der von $\mathrm{C}_{2} \mathrm{HF}_{5}$. Um die vollständige Reaktion zu beschreiben wurde ein Mechanismus mit 68 Reaktionsschritten postuliert [3], mit den ersten Schritten:

$$
\begin{aligned}
& \mathrm{C}_{3} \mathrm{~F}_{7} \mathrm{H}+(\mathrm{M}) \longrightarrow \mathrm{C}_{3} \mathrm{~F}_{6}+\mathrm{HF}+(\mathrm{M}) \\
& \mathrm{C}_{3} \mathrm{~F}_{7} \mathrm{H}+(\mathrm{M}) \longrightarrow \mathrm{CF}_{3} \mathrm{CHF}+\mathrm{CF}_{3}+(\mathrm{M})
\end{aligned}
$$

In der hier vorgestellten Arbeit kann der schnelle Zerfall von $\mathrm{C}_{3} \mathrm{~F}_{6}$, gefolgt von der Reaktion (1.3), einen Einblick in Reaktion (1.4) geben 


$$
\mathrm{C}_{3} \mathrm{~F}_{6}+(\mathrm{M}) \longrightarrow \mathrm{C}_{2} \mathrm{~F}_{4}+\mathrm{CF}_{2}+(\mathrm{M})
$$

Die folgenden Kapitel erläutern den experimentellen Aufbau und beschreiben die Auswertung der gemessenen Rohdaten. Darauf folgt die Analyse und Diskussion der erhaltenen Resultate. 


\section{Kapitel 2}

\section{Experimentelle Technik}

\subsection{Die Stoßwellentechnik}

Die gut entwickelte Stoßwellentechnik [14-17] bietet die Gelegenheit die Kinetik von Elementarreaktionen über einen großen Temperatur- und auch Druckbereich zu untersuchen. Im Folgenden wird auf die wesentlichen Eigenschaften von Stoßwellen und der in dieser Arbeit verwendeten Technik eingangen.

Stoßwellen können in allen Phasen erzeugt werden und breiten sich normalerweise kugelförmig aus. Durch die Nutzung eines Rohres wird eine eindimensionale Ausbreitungsrichtung erzwungen. Im hier genutzen gasförmigen Medium kommt es in der Stoßfront zu einem Druckanstieg und einem Temperatursprung unterhalb einer Mikrosekunde. Dabei wird in der vorliegenden Arbeit ein Druckbereich von $[\mathrm{Ar}]=2 \cdot 10^{-5}$ bis $10 \cdot 10^{-5} \mathrm{~mol} / \mathrm{cm}^{3}$ (entspricht $3-12 \mathrm{bar}$ ) und ein Temperaturbereich von 1200 - $2000 \mathrm{~K}$ abgedeckt.

Für die durchgeführten Experimente werden Stoßwellen in einem Rohr mit konstantem Durchmesser erzeugt. Das Rohr wird durch eine Aluminiummembran in einen Hoch- und einen Niederdruckteil aufgeteilt. Der Niederdruckteil enthält das Testgasgemisch, während in den Hochdruckteil Wasserstoff als Treibgas gefüllt wird bis die Membran birst, woraufhin sich die Stoßwelle bildet.

Um die Entwicklung einer Stoßwelle zu verstehen, dient ein beschleunigter, mechanischer Kolben als gutes Beispiel. Der Kolben bewegt sich vom Hoch- in den Niederdruckteil, wobei die davorlaufende Verdichtungswelle das Testgas adiabatisch aufheizt und sich dabei die Schallgeschwindigkeit erhöht. Diese ist durch die Beziehung 


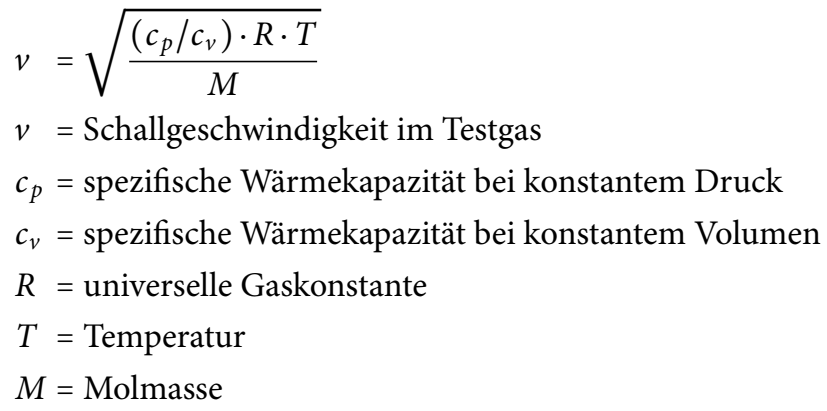

gegeben. Durch die Erhöhung der Schallgeschwindigkeit können nachfolgende Verdichtungswellen die vorangegangenen einholen und somit das Testgas weiter erwärmen. Nach etwa zehn Rohrdurchmessern kommt es zur Bildung einer ebenen Stoßfront. Diese breitet sich im Verhältnis zum ruhenden, kalten Gas mit Überschallgeschwindigkeit aus. Die Dicke der Stoßfront beträgt einige freie Weglängen innerhalb derer Druck, Dichte und Temperatur stark ansteigen. Die geringe Dicke bedingt, dass die Endwerte nach weniger als $100 \mathrm{~ns}$ erreicht werden. Damit ist es möglich, auch kurzlebige Spezies, zum Beispiel radikalische Zwischenprodukte, zu untersuchen.

Am Ort der Stoßfront existiert ein Brechungsindexgradient, der eine Ablenkung des Analysenlichts bewirkt. Dies äußert sich in einer Schliere, d.h. einem scharfen Peak im Absorptionssignal. Dieser wird stets detektiert, startet die Beobachtungszeit am Anfang der Reaktion und dient weiterhin als Markierung für den Zeitpunkt des Temperatursprungs.

Bei allen vorliegenden Experimenten wird ein Stoßrohr mit planarer Endplatte verwendet, an der die einfallende Welle reflektiert wird. Nach der Reflektion der Welle erfolgt ein zweiter Temperatur-, Dichte- und Drucksprung. Es besteht nun die Möglichkeit, die Reaktion in der einfallenden oder in der reflektierten Welle zu detektieren. Dabei ist darauf zu achten, dass die korrekten Dichteverhältnisse $\left(\rho_{2} / \rho_{1}\right.$ bzw. $\left.\rho_{5} / \rho_{1}\right)$ für die jeweilige Welle in den Berechnungen berücksichtigt werden.

Die Untersuchung der einfallenden Welle wird zeitlich durch das Eintreffen der reflektierten Welle begrenzt. Der Zeitrahmen von ca. $1.5 \mathrm{~ms}$ für die reflektierte Welle ergibt sich aus dem Eintreffen von Verdünnungswellen, welche sich rückwärts in das Treibgas ausbreiten und an der Endplatte ebenfalls reflektiert werden.

Ein großer Vorteil der verwendeten Stoßwellentechnik ist neben der geringen benötigten Substanzmenge, die einfache Variation der Reaktionsbedingungen. Durch Nutzung verschieden starker Aluminiummembranen wird der Druck und durch 
Anpassen des Vordrucks im Niederdruckteil die Temperatur über einen weiten Bereich geregelt. Die Methode ist daher für die vorliegenden Untersuchungen von Gasphasenreaktionen gut geeignet.

\subsubsection{Berechnung der Zustandsdaten}

Zur richtigen Interpretation der erhaltenen Daten aus einem Experiment ist es unabdingbar, die in der Stoßwelle herrschenden Bedingungen, also Druck, Dichte und Temperatur, zu kennen. $\mathrm{Zu}$ unterscheiden sind hierbei immer die Bedingungen in der einfallenden und in der reflektierten Welle.

Man bedient sich dabei der Rankine-Hugoniot-Gleichungen, welche sich aus der Anwendung der Erhaltungssätze für Masse, Impuls und Energie auf eine eindimensionale Strömung mit konstanter Ausbreitungsgeschwindigkeit ergeben. Die Gleichungen beschreiben die Zustandsdaten auf beiden Seiten der Stoßwelle.

Energieerhaltung:

$$
H_{1}+\frac{u_{1}^{2}}{2}=H_{2}+\frac{u_{2}^{2}}{2}
$$

Massenerhaltung:

$$
\rho_{1} u_{1}=\rho_{2} u_{2}
$$

Impulserhaltung:

$$
p_{1}+\rho_{1} u_{1}^{2}=p_{2}+\rho_{2} u_{2}^{2}
$$

Hierbei ist $u$ die Strömungsgeschwindigkeit relativ zur Stoßfront, $H$ die massenspezifische Enthalpie, $p$ der hydrostatische Druck und $\rho$ die Dichte.

Im Folgenden wird die übliche Konvention der Bezifferung genutzt. Der Index 1 bezeichnet Zustandsdaten vor der Stoßfront, Zustandsdaten nach der einfallenden Welle erhalten den Index 2 und diejenigen nach der reflektierten Welle den Index 5.

Da in allen Experimenten hochverdünnte Mischungen aus einem Edelgas (Argon) und dem Testgas genutzt werden, kann das Gasgemisch als ideales Gas mit konstanter spezifischer Wärme behandelt werden. Es gilt:

$$
p_{2}=\rho_{2} \cdot R \cdot T_{2}
$$




$$
H_{2}-H_{1}=c_{p} \cdot\left(T_{2}-T_{1}\right)
$$

Aus den Gleichungen (2.2) bis (2.6) ergibt sich:

$$
\begin{aligned}
& \frac{p_{2}}{p_{1}}=\frac{2 \kappa \cdot M_{1}^{2}-(\kappa-1)}{(\kappa+1)} \\
& \frac{\rho_{2}}{\rho_{1}}=\frac{M_{1}^{2} \cdot(\kappa+1)}{(\kappa-1) \cdot M_{1}^{2}+2} \\
& \frac{T_{2}}{T_{1}}=\frac{p_{2}}{p_{1}} \cdot \frac{\rho_{2}}{\rho_{1}}
\end{aligned}
$$

mit $\kappa=\frac{c_{p}}{c_{v}}$ und der Machzahl $M_{1}=\frac{u_{1}}{V_{1}}$

Aus den Anfangsbedingungen $\left(p_{1}, \rho_{1}, T_{1}\right)$ und den gemessenen Laufzeiten der Stoßwelle, d.h. der Stoßwellengeschwindigkeit, lassen sich mit den Gleichungen (2.7) bis (2.9) die Zustandsdaten nach der einfallenden Welle berechnen.

Wird die Annahme getroffen, dass sich das Testgas nach Passieren der reflektierten Welle wieder in Ruhe befindet, können mit folgenden Formeln die Zustandsdaten nach der reflektierten Welle erhalten werden:

$$
\begin{aligned}
& \frac{p_{5}}{p_{1}}=\left[\frac{\left(\frac{3 \kappa-1}{\kappa-1}\right) M_{1}^{2}-2}{M_{1}^{2}+\left(\frac{2}{\kappa-1}\right)}\right] \cdot\left[\frac{\left(\frac{2 \kappa}{\kappa-1}\right) M_{1}^{2}-1}{\left(\frac{\kappa+1}{\kappa-1}\right)}\right] \\
& \frac{\rho_{5}}{\rho_{1}}=\left[\frac{M_{1}^{2} \cdot\left(\frac{\kappa+1}{\kappa-1}\right)}{M_{1}^{2}+\left(\frac{2}{\kappa-1}\right)}\right] \cdot\left[\frac{\left(\frac{2 \kappa}{\kappa-1}\right) \cdot M_{1}^{2}-1}{M_{1}^{2}+\left(\frac{3-\kappa}{\kappa-1}\right)}\right] \\
& \frac{T_{5}}{T_{1}}=\frac{\left[\left(\frac{3 \kappa-1}{\kappa-1}\right) \cdot M_{1}^{2}-2\right] \cdot\left[2 M_{1}^{2}+\left(\frac{3-\kappa}{\kappa-1}\right)\right]}{\left(\frac{\kappa+1}{\kappa-1}\right)^{2} \cdot M_{1}^{2}}
\end{aligned}
$$


Mit den beschriebenen Gleichungen können alle relevanten Zustandsdaten für das, als ideal angenommene, Testgasgemisch temperaturunabhängig berechnet werden. Führt man nun verfügbare thermodynamische Daten [18] für das jeweilige zu untersuchende Gas ein, so können die erreichten Zustandsdaten temperaturabhängig und somit explizit abgeleitet werden.

\subsubsection{Experimenteller Aufbau}

In der vorliegenden Arbeit wird ein Aluminiumstoßrohr mit einem Innendurchmesser von $100 \mathrm{~mm}$ und einer Wandstärke von $10 \mathrm{~mm}$ verwendet. Die Gesamtlänge beträgt $6,95 \mathrm{~m}$, wovon $2,80 \mathrm{~m}$ auf den Niederdruckteil und 4,15 $\mathrm{m}$ auf den Hochdruckteil entfallen. Die Trennung von Hoch- und Niederdruckteil erfolgt durch Aluminiummembranen verschiedener Stärke $(0,4 \mathrm{~mm}-0,01 \mathrm{~mm})$, die in einen mit O-Ringen (FKM) abgedichteten Edelstahlflansch mit Schnellverschluss eingespannt werden. Während der Niederdruckteil fest auf zwei Betonsockeln montiert ist, lagert der Hochdruckteil auf Rollen, so dass er zum Reinigen und Einspannen der Membran vom Rest separiert werden kann. Das Analysenlicht gelangt nach Durchgang durch eine Linse der Brennweite $50 \mathrm{~mm}$ und eine Schlitzblende (Breite $1 \mathrm{~mm}$ ) durch zwei planare Tetrasil-Fenster (14,2 mm nutzbarer Durchmesser) durch das Stoßrohr. Die Fenstereinlassungen befinden sich $45 \mathrm{~mm}$ vor der Endplatte. Danach erfolgt die Führung des Lichtstrahls durch eine weitere Linsen- (Brennweite $100 \mathrm{~mm}$ ) und Schlitzblendenkombination $(1 \mathrm{~mm})$ um von dort aus in den Monochromator (Zeiss MQ3) zu gelangen. Nach Auswahl der geeigneten Wellenlänge wird das Licht auf einen Photomultiplier (PMT, Hamamatsu 1P28) übertragen.

Die Endplatte selbst wird durch einen O-Ring (FKM) gedichtet und mit vier Bolzen am Aluminiumrohr befestigt. Nach jedem Einzelschuss wird die Platte abgenommen, die Reste der Aluminiummembran ausgeblasen und die Fenster werden mit Aceton p.a. gereinigt.

Das Stoßrohr wird mittels Drehschieberpumpe (Pfeiffer Duo016B) auf Drücke kleiner $3 \cdot 10^{-3}$ mbar evakuiert. Vor jeder Messung wird der Niederdruckteil zusätzlich mit einer Turbomolekularpumpe (Balzers Typ TPU 240, Vorpumpe Trivac D16) bis zu Drücken im Bereich von $10^{-5}$ bis $10^{-7}$ mbar evakuiert.

Nach Evakuierung des Rohrs wird das Testgasgemisch aus den vorher befüllten Vorratsbehältern über ein Faltenbalgventil am Ende des Niederdruckteils bis zum gewünschten Vordruck eingefüllt. Hierfür werden Absolutdruck-Messgeräte vom Typ MKS 627D (Messbereich 0 - 20 mbar, bzw. 0 - 1000 mbar) genutzt. Wasserstoff (3.0), als Treibgas, wird über ein Magnetventil bis zum Bersten der Membran eingelassen. 


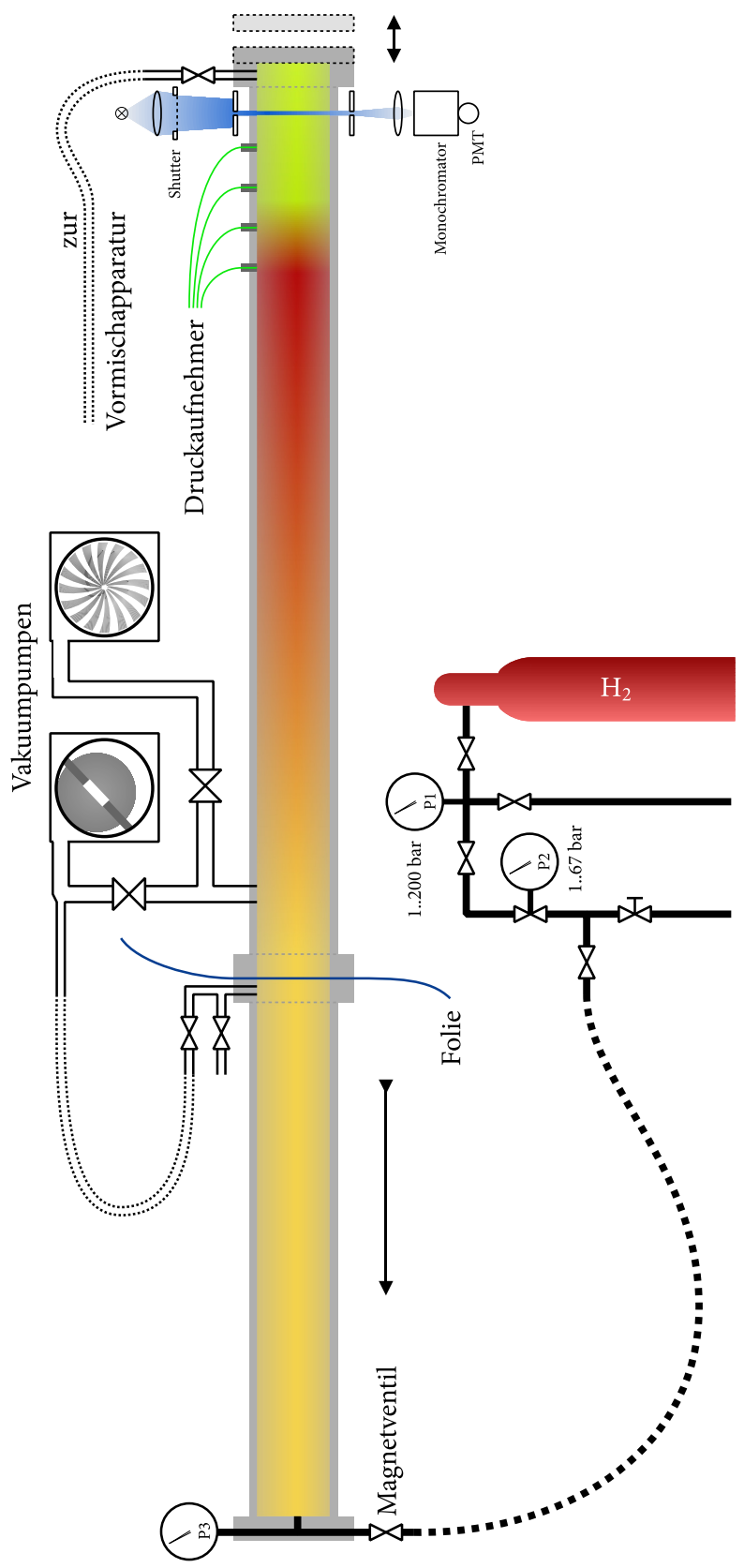

Abb. 2.1: Aufbau des Stoßwellenexperiments 


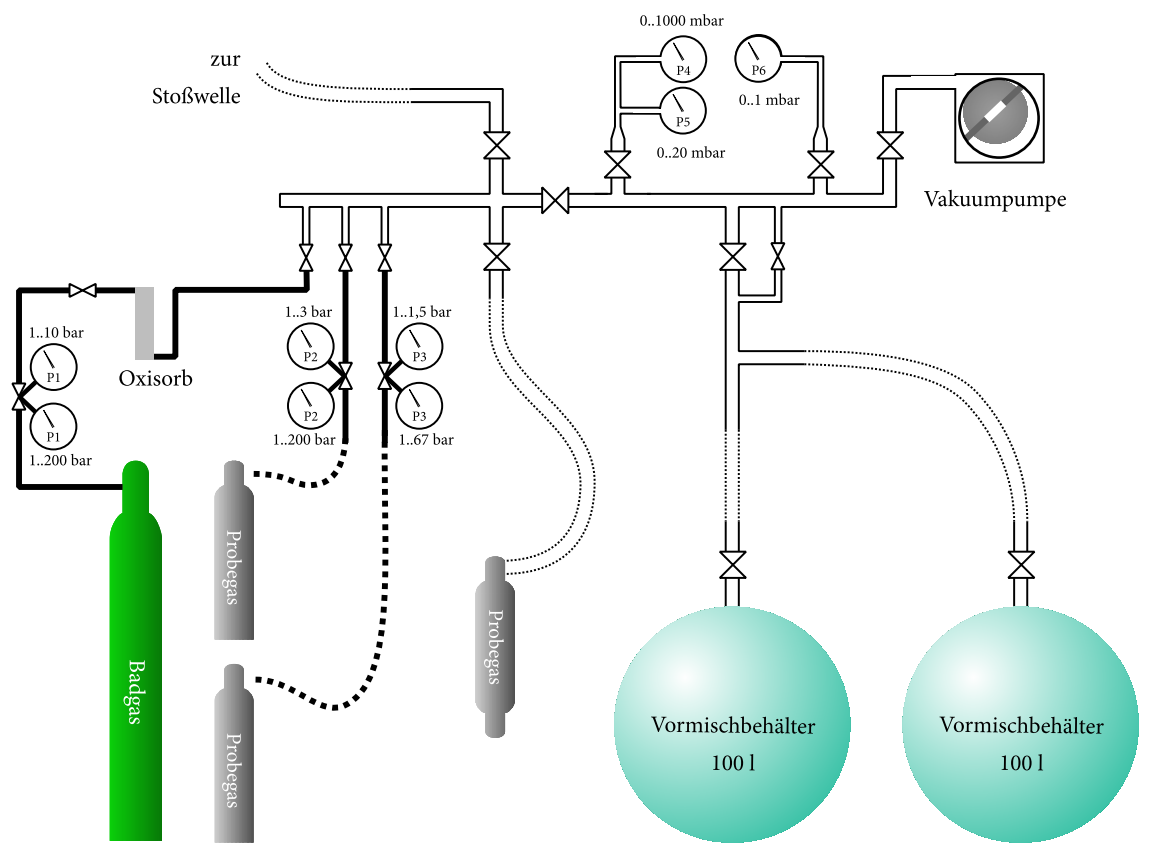

Abb. 2.2: Aufbau der Vormischapparatur

Zur Bestimmung der Geschwindigkeit der Stoßwelle wurden für diese Arbeit die bisher genutzten Platinstreifen durch vier piezoelektrische Drucksensoren (Kistler Typ 603B) ersetzt. Der durch die Stoßwelle verursachte Druckanstieg wird über die Membran auf einen Quarzkristall, welcher als Messelement fungiert, übertragen und in eine elektrische Ladung (in pC) umgewandelt. Die Drucksensoren zeichnen sich durch ihre besondere Dichtigkeit aus. Membran und Sensorgehäuse sind aus rostfreiem Stahl gefertigt und hermetisch und bündig verschweißt. Der dazugehörige keramische Isolator ist dabei so dicht in den Stecker integriert, dass eine hohe Dichtigkeit gewährleistet werden kann. Weiterhin sind die piezoelektrischen Quarze mit einer seismischen Masse verbaut, was die Beschleunigungsempfindlichkeit zum großen Teil kompensiert. Dadurch sind die Sensoren besonders für den Einsatz bei hohen Druckschwankungen mit kurzer Anstiegszeit in vibrierenden Objekten geeignet und daher ein hervorragendes Bauteil für die Laufzeitmessung innerhalb eines Stoßrohres. Die vier Drucksensoren werden mit passenden Halterungen in die vorhandenen Öffnungen im Stoßrohr eingelassen. 


\subsubsection{Signalverarbeitung}

Zur Signalaufbereitung kommen vier passende Ladungsverstärker (Kistler Typ 5018A) zum Einsatz, welche das ankommende Signal der Drucksensoren auf einen Bereich von -8 bis $10 \mathrm{~V}$ verstärken. Die Ausgänge der Ladungsverstärker sind mit einer Komparatorschaltung gekoppelt. Diese generiert aus dem verstärkten Signal der Drucksensoren eine Serie von vier zu unterscheidenden TTL-Pulsen, welche an einem digitalen Eingang der Messkarte (Spektrum M3i.4111-Exp 2 Kanal 14bit A/D, Transientenrekorder) zeitsynchron mit dem analogen Signal des Photomultipliers aufgezeichnet werden. Das Signal des ersten Drucksensors dient hierbei zusätzlich als Haupttrigger für eine Messkarte und startet die Messung.

Unabhängig von der Zeitbasis der Messwertaufnahme werden drei Messkartenausgänge für unterschiedliche Steuerungen einzelner Komponenten des experimentellen Aufbaus genutzt. Ein Ausgang öffnet und schliesst den Shutter vor der Lampe (Osram XBO 150 W/4 Suprasil). Dadurch wird eine Verlängerung der Lebensdauer des PMT erreicht, da nur während einer Messung Licht einfällt. Weiterhin wird die Steuerung für das Magnetventil freigeschaltet. Dies dient der Sicherheit, da so sichergestellt werden kann, dass nur unter hergestellter Messbereitschaft der Hochdruckteil mit $\mathrm{H}_{2}$ befüllt werden kann. Der verbliebene Ausgang aktiviert die Ladungsverstärker vor einer Messung, so dass diese ausschließlich während einer Messung Impulse der Drucksensoren aufnehmen.

Die Signale der vier Ladungsverstärker werden parallel an ein Oszilloskop (Tektronix TDS 3014B) gesendet, mit dem die Triggerschwelle in Abhängigkeit von Membrandicke und Vordruck erstmalig festgelegt werden kann. Weiterhin können die Druckverläufe direkt nachvollzogen werden, um etwaige Fehler zu beobachten und beheben zu können.

Mit dem PMT wird die UV-Transmission von $\mathrm{CF}_{2}$ nach Durchgang durch den Monochromator aufgenommen und verstärkt. Daraufhin erfolgt die Einspeisung des analogen Signals in die Messkarte. Dabei wird eine theoretisch maximale Auflösung von 13 bit genutzt.

Die von Lars Sölter programmierte Messsoftware stützt sich auf Agilent VEE und wird in dieser Arbeit unverändert benutzt. Die Software verarbeitet, zusammen mit den eingegebenen Zustandsdaten des ruhenden Systems, die ankommenden Daten der Messkarte und berechnet daraus direkt die Zustandsdaten in der einfallenden und reflektierten Welle. 


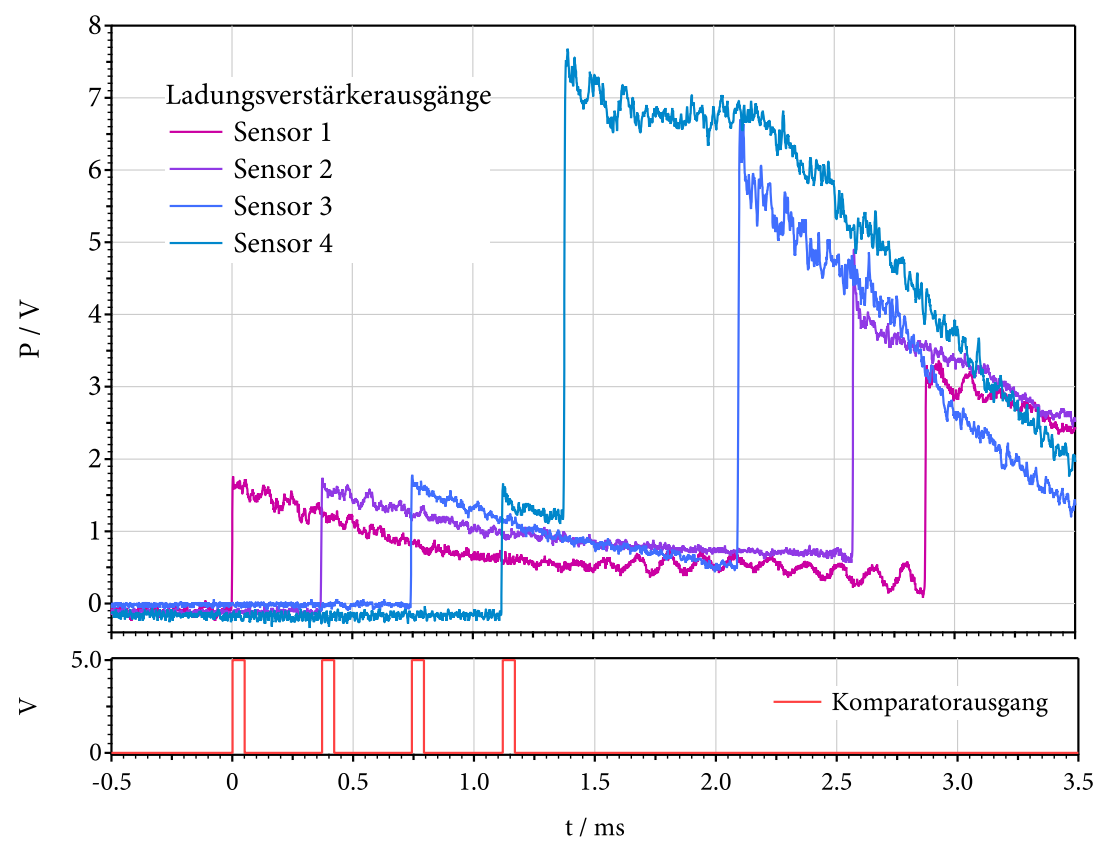

Abb. 2.3: Druckaufnehmersignal und dazugehöriges Komparator-Ausgangssignal

Tab. 2.1: Verwendete Chemikalien

\begin{tabular}{llcccc}
\hline Substanz & Hersteller & Kurzform & CAS Nr. & Kurzzeichen & Reinheit [\%] \\
\hline Pentafluorethan & $\mathrm{abcr}$ & $\mathrm{C}_{2} \mathrm{HF}_{5}$ & $354-33-6$ & $\mathrm{R}-125$ & 98 \\
Heptafluorpropan & $\mathrm{abcr}$ & $\mathrm{C}_{3} \mathrm{HF}_{7}$ & $431-89-0$ & R-227ea & 99.5 \\
Trifluormethan & Linde & $\mathrm{CHF}_{3}$ & $75-46-7$ & - & 99.9999 \\
Tetrafluorethen & Dyneon & $\mathrm{C}_{2} \mathrm{~F}_{4}$ & $116-14-3$ & - & $>97$ \\
\hline Wasserstoff & Linde & $\mathrm{H}_{2}$ & $1333-74-0$ & & 99,9 \\
Argon & Linde & $\mathrm{Ar}$ & $7440-37-1$ & & 99,9999 \\
\hline
\end{tabular}




\subsubsection{Verwendete Chemikalien}

Die verwendeten Substanzen werden von der Firma Dyneon GmbH zur Verfügung gestellt. Argon und Wasserstoff sind kommerziell erhältliche Standardgase. Argon zur Herstellung der Testgasmischungen wird mit einer Oxysorbkartusche von Sauerstoff und Wasser befreit. Wasserstoff kann hingegen ohne weitere Aufreinigung benutzt werden. 


\section{Kapitel 3}

\section{Auswertung}

\subsection{Allgemeines}

In der Vergangenheit wurden in dieser Arbeitsgruppe bereits die thermischen Zerfälle von $\mathrm{CHF}_{3}$ [19], $\mathrm{C}_{2} \mathrm{~F}_{6}$ [20] sowie $\mathrm{C}_{2} \mathrm{~F}_{4}$ [13] untersucht. Diese Moleküle zerfallen ebenso wie das Pentafluorethan und $2 \mathrm{H}$-Heptafluorpropan $\mathrm{zu} \mathrm{CF}_{2}$. Es ist daher naheliegend auch höhere Homologe zu bearbeiten und die Resultate mit früheren Ergebnissen zu vergleichen.

\subsubsection{Experimentelle Bedingungen}

Der Zerfall von Pentafluorethan und 2H-Heptafluorpropan $\mathrm{zu} \mathrm{CF}_{2}$ wird in einfallenden und reflektierten Stoßwellen in einem Temperaturbereich von 1200 - $2000 \mathrm{~K}$ bei drei verschiedenen Mischungsverhältnissen (etwa 1000 ppm, 500-600 ppm, 70 ppm) untersucht. Unterschiedliche Drücke erreicht man durch das Benutzen verschieden dicker Folien und durch Messungen entweder in der einfallenden oder reflektierten Welle. Die Badgaskonzentration liegt zwischen $1.8 \cdot 10^{-5}$ und $9.8 \cdot 10^{-5} \mathrm{~mol} / \mathrm{cm}^{3}$ in der reflektierten Welle. Dies entspricht Drücken von etwa 3-12 bar.

\subsubsection{Der Absorptionskoeffizient}

Ein erster Schritt zur Auswertung der gemessenen Daten ist die Bestimmung des Absorptionskoeffizienten $\epsilon$ von $\mathrm{CF}_{2}$. Darauf aufbauend können im Folgenden andere Parameter, wie etwa Geschwindigkeitskonstanten oder Produktausbeuten berechnet werden. In dieser Arbeit wird deshalb der Absorptionskoeffizient von $\mathrm{CF}_{2}$ bei $\lambda=$ $248 \mathrm{~nm}$ in Abhängigkeit der Temperatur mittels des Lambert-Beer'schen Gesetzes verifiziert.

Um einen möglichst genauen Wert zu erhalten, werden dabei ausschließlich experimentelle Messungen ausgewählt, bei denen gesichert ist, dass das Ausgangsmolekül 
vollständig zu $\mathrm{CF}_{2}$ zerfällt. Als Ausgangsmoleküle dienen $\mathrm{C}_{2} \mathrm{~F}_{4}$ und $\mathrm{CHF}_{3}$. Die Messungen von $\mathrm{C}_{2} \mathrm{HF}_{5}$ und $\mathrm{C}_{3} \mathrm{HF}_{7}$ werden nicht berücksichtigt, da der Zerfall nicht in einem Schritt direkt erfolgt und somit das Maximum der erreichten Absorption nicht zur Ermittlung des Absorptionskoeffizienten herangezogen werden kann. Zur Bestimmung des Absorptionsmaximums wird ein Polynomfit zur Glättung über einen Ausschnitt der Messung gelegt und das Maximum abgelesen. (Abb. 3.1) Die für den Absorptionskoeffizienten erhaltenen Werte werden mit verfügbaren Daten aus der Literatur [21-26] verglichen. Literaturdaten, die in einem anderen Druckoder Wellenlängenbereich liegen, werden entsprechend umgerechnet. Dabei wird zunächst das bekannte Sulzer-Wieland Modell [27] eingesetzt, d.h. $\epsilon$ dargestellt durch

$$
\begin{aligned}
& \epsilon(\lambda, T) \approx \epsilon_{0}^{m} \prod_{i=1}^{s} \sqrt{\tanh \left(\frac{\theta_{0}}{2 T}\right)} \cdot \exp \left(-\sum_{i=1}^{s} \tanh \left(\frac{\theta_{0}}{2 T}\right)\left(\frac{\lambda-\lambda_{0}}{\Delta \lambda_{0 i}}\right)^{2}\right) \\
& \epsilon(\lambda, T)=1.07 \cdot 10^{7} \mathrm{~cm}^{2} \mathrm{~mol}^{-1} \\
& \lambda_{0} \quad=40300 \mathrm{~cm}^{-1} \\
& \Delta \lambda_{0 i} \quad=1100 \mathrm{~cm}^{-1} \\
& \theta_{0 i} \quad=960 \mathrm{~K} \\
& s \quad=2
\end{aligned}
$$

Die Sulzer-Wieland Anpassung gibt die Werte von Carlson [26 und Battin-Leclerc [21] gut wieder. Allerdings passen die Daten von Schug und Wagner [25], sowie die in dieser Arbeit gemessenen Werte weniger gut zu diesem theoretischen Ansatz. Daher wird als Alternative eine Gauss-Anpassung an die Messdaten vorgenommen, die die experimentellen Ergebnisse am besten abbildet (Abb. 3.2). Es gilt dabei folgende Abhängigkeit:

$$
\epsilon(\lambda=248 \mathrm{~nm}, T) \approx 3.3510^{6}+4.610^{7} \cdot \mathrm{e}^{-\left(\frac{T+1457 \mathrm{~K}}{1272 \mathrm{~K}}\right)^{2}}-245 \cdot(T / \mathrm{K})
$$

Mit dem dargestellten Absorptionskoeffizienten $\epsilon(\lambda=248 \mathrm{~nm}, T)$ (Abb. 3.2 werden alle weiteren Rechnungen ausgeführt.

\subsubsection{Signalauswertung}

Zur Beobachtung der Reaktionskinetik von $\mathrm{C}_{2} \mathrm{HF}_{5}$ und $\mathrm{C}_{3} \mathrm{HF}_{7}$ wird der im Experimentalteil beschriebene optische Aufbau genutzt. Die Abbildungen 3.3, 3.4 und 3.5 


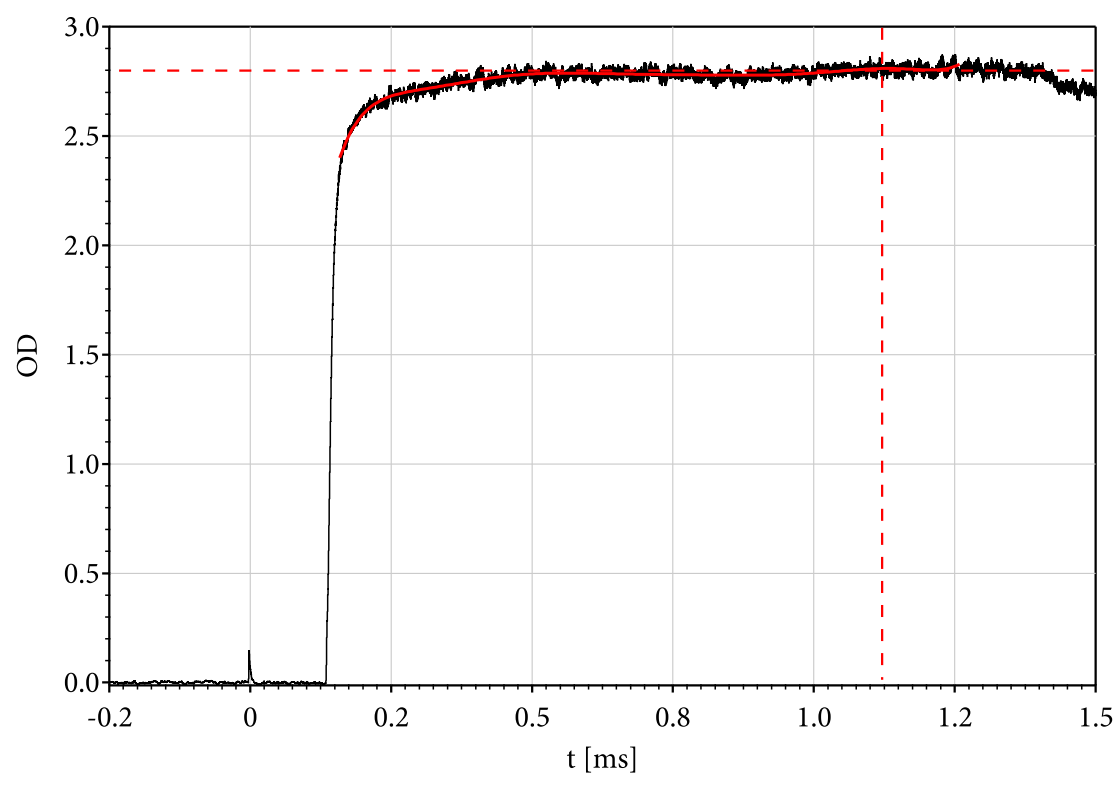

Abb. 3.1: Bestimmung des Absorptionsmaximums

Tab. 3.1: Absorptionskoeffizienten

\begin{tabular}{cc|cc|cc}
\hline & & $|\mathbf{2 2}| \mathbf{2 4} \mid$ & $\mid$ & \\
$\epsilon\left(10^{6} \mathrm{~cm}^{3} \mathrm{~mol}^{-1}\right)$ & $\mathrm{T} / \mathrm{K}$ & $\epsilon\left(10^{6} \mathrm{~cm}^{3} \mathrm{~mol}^{-1}\right)$ & $\mathrm{T} / \mathrm{K}$ & $\epsilon\left(10^{6} \mathrm{~cm}^{3} \mathrm{~mol}^{-1}\right)$ & $\mathrm{T} / \mathrm{K}$ \\
\hline 9.97 & 295 & 1.709 & 2878 & 3.00 & 1400 \\
9.75 & 323 & 2.252 & 2085 & 3.00 & 2000 \\
8.44 & 373 & 2.717 & 1804 & 3.00 & 2700 \\
7.00 & 473 & & & & \\
6.29 & 573 & & & & \\
5.34 & 673 & & & & \\
4.42 & 873 & & & & \\
\hline
\end{tabular}




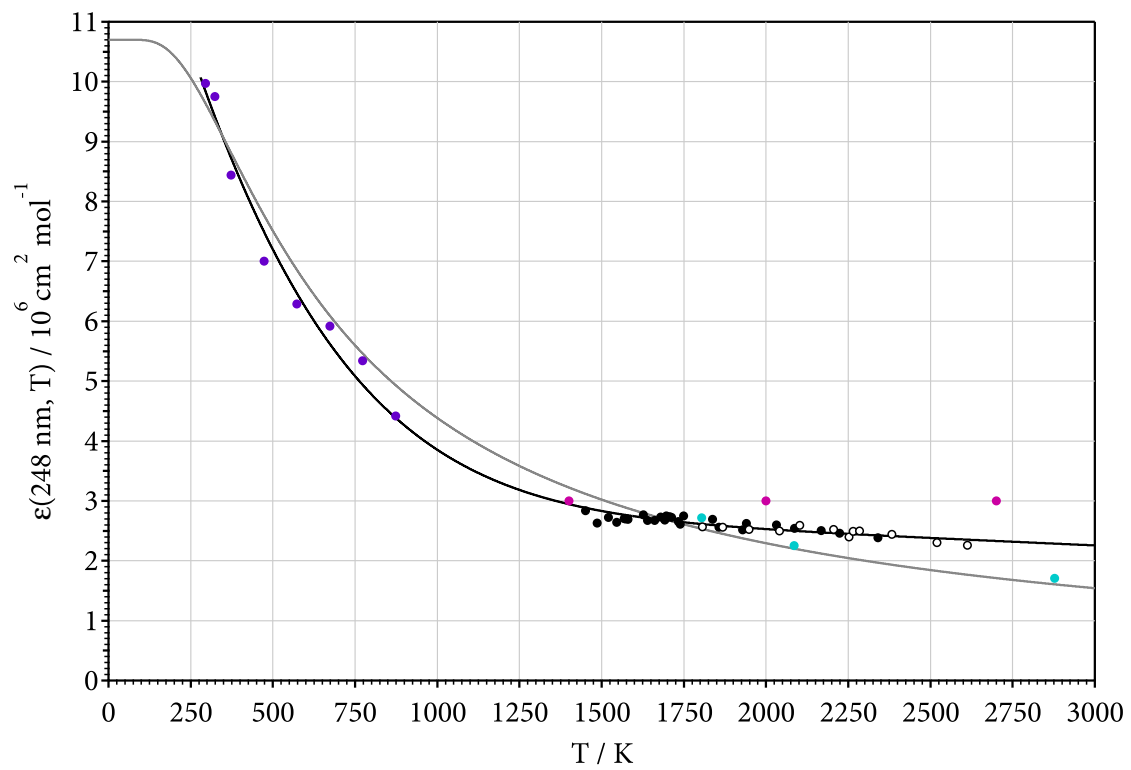

Abb. 3.2: Absorptionskoeffizient von $\mathrm{CF}_{2} \cdot \bullet: \mathrm{C}_{2} \mathrm{~F}_{4}$, diese Arbeit; $\circ: \mathrm{CHF}_{3}$, diese Arbeit; $\bullet$ : Schug und Wagner [25]; : Modica [22,-24]; •: Battin-Leclerc [21]; graue Linie: SulzerWieland-Anpassung; schwarze Linie: Gauss-Anpassung

zeigen typische Einzelschussergebnisse von Pentafluorethan bei verschiedenen Temperaturen in der reflektierten Welle. Aufgetragen ist hierbei die optische Dichte (OD) gegen die Zeit. Vor jedem Schuss werden drei Dunkelmessungen vorgenommen und gemittelt, daraus resultiert die Intensität $\mathrm{I}_{\text {dunkel }}$. Das gemittelte Signal wird von der folgenden Messung abgezogen. Zur Ermittlung der Intentsität des Analysenlichts $\mathrm{I}_{0}$ wird das Signal im Zeitraum zwischen dem Anfang der Messperiode und dem ersten Triggersignal (einfallende Welle) gemittelt. Nach dem Gesetz von Lambert-Beer erhält man die optische Dichte wie folgt:

$$
O D=\log \left(\frac{I-I_{\text {dunkel }}}{I_{0}-I_{\text {dunkel }}}\right)
$$

Im Folgenden werden Absorptions-Zeit-Profile von Zerfallsexperimenten betrachtet. Die Abbildungen (3.3. 3.4 und 3.5) zeigen deutlich die Schlieren der einfallenden und reflektierten Welle. Der Nullpunkt der Zeit wird auf das Maximum der Schlierensignale der einfallenden oder reflektierten Welle gelegt, je nachdem hinter welcher 
Front die Reaktion untersucht wird. In Abhängigkeit von der Temperatur kommt es hinter der einfallenden oder reflektierten Welle zu einem Anstieg der Absorption durch sich bildendes $\mathrm{CF}_{2}$. Bei Temperaturen von $T_{5}=1200-2000 \mathrm{~K}$ finden die Reaktionen von $\mathrm{C}_{2} \mathrm{HF}_{5}$ und $\mathrm{C}_{3} \mathrm{HF}_{7}$ ausschließlich hinter der reflektierten Welle statt. Um Absorptionssignale von $\mathrm{CF}_{2}$ in der einfallenden Welle zu erhalten, muss $T_{5}$ deutlich über $2000 \mathrm{~K}$, bzw. $T_{2}$ über $1200 \mathrm{~K}$ liegen.

Abb. 3.3 zeigt ein Absorptions-Zeit Profil von $\mathrm{C}_{2} \mathrm{HF}_{5}$ bei einer Temperatur von $1269 \mathrm{~K}$ und einer Reaktandenkonzentration von 1000 ppm. Die Absorption des $\mathrm{CF}_{2}$ beginnt hinter der reflektierten Welle und steigt, bedingt durch die niedrige Temperatur, nur langsam an, ohne im Beobachtungszeitraum ein Maximum zu erreichen.

Hingegen beschreibt Abb. 3.4 ein Absorptions-Zeit-Profil von $\mathrm{C}_{2} \mathrm{HF}_{5}$ bei einer Temperatur von $1596 \mathrm{~K}$ und einer Reaktandenkonzentration von 1000 ppm. Die Absorption von $\mathrm{CF}_{2}$ beginnt hier ebenfalls hinter der reflektierten Welle, steigt jedoch bedeutend schneller an und erreicht ein beinahe konstantes Maximum.

Bei hohen Temperaturen wie in Abb. 3.5 bei $2049 \mathrm{~K}$ und 1000 ppm erfolgt die Absorption sehr schnell hinter der reflektierten Welle, weshalb der Anstieg des Signals fast senkrecht erscheint. Die Bildung des $\mathrm{CF}_{2}$ durchläuft schon nach kurzer Zeit ein Absorptionsmaximum um in eine kurze Sättigung überzugehen. Danach kommt es nach etwa $0.75 \mathrm{~ms}$ zu einem weiteren kleinen Anstieg des Absorptionssignals, d.h. es wird auf einer längeren Zeitskala neues $\mathrm{CF}_{2}$ verzögert gebildet.

Abb. 3.6 zeigt die $\mathrm{CF}_{2}$-Bildung nach der einfallenden Welle bei einer Temperatur von $2017 \mathrm{~K}$ und 1000 ppm. Die Radikalbildung erfolgt schnell und geht in eine Sättigung über, bis zum Eintreffen der reflektierten Welle. Danach ist ein rapider Abfall zu beobachten, da es durch den Temperatursprung zu sehr schnellen Folgereaktionen kommt und die Bildung von $\mathrm{CF}_{2}$ abgeschlossen ist.

\subsection{Detailauswertung}

\subsubsection{Bestimmung der Ausbeute}

Der erste Schritt zur Auswertung der gemessenen Signale ist die Bestimmung der maximalen $\mathrm{CF}_{2}$-Ausbeute. Der erhaltene Maximalwert der beobachteten optischen Dichte $O D_{\max }$ wird mit dem Absorptionskoeffizienten $\epsilon(\lambda=248 \mathrm{~nm}, T)$ verglichen, wodurch sich die Ausbeute an $\mathrm{CF}_{2}$ pro $\mathrm{C}_{2} \mathrm{HF}_{5}-$ Molekül (bzw. pro $\mathrm{C}_{3} \mathrm{HF}_{7}-$ Molekül) bestimmen lässt.

Dabei zeigt sich, dass unabhängig von der Reaktandenkonzentration ein $\mathrm{C}_{2} \mathrm{HF}_{5}$ Molekül zu zwei $\mathrm{CF}_{2}$ (bzw. ein $\mathrm{C}_{3} \mathrm{HF}_{7}-$ Molekül zu drei $\mathrm{CF}_{2}$ ) zerfällt. Die Ausbeute 


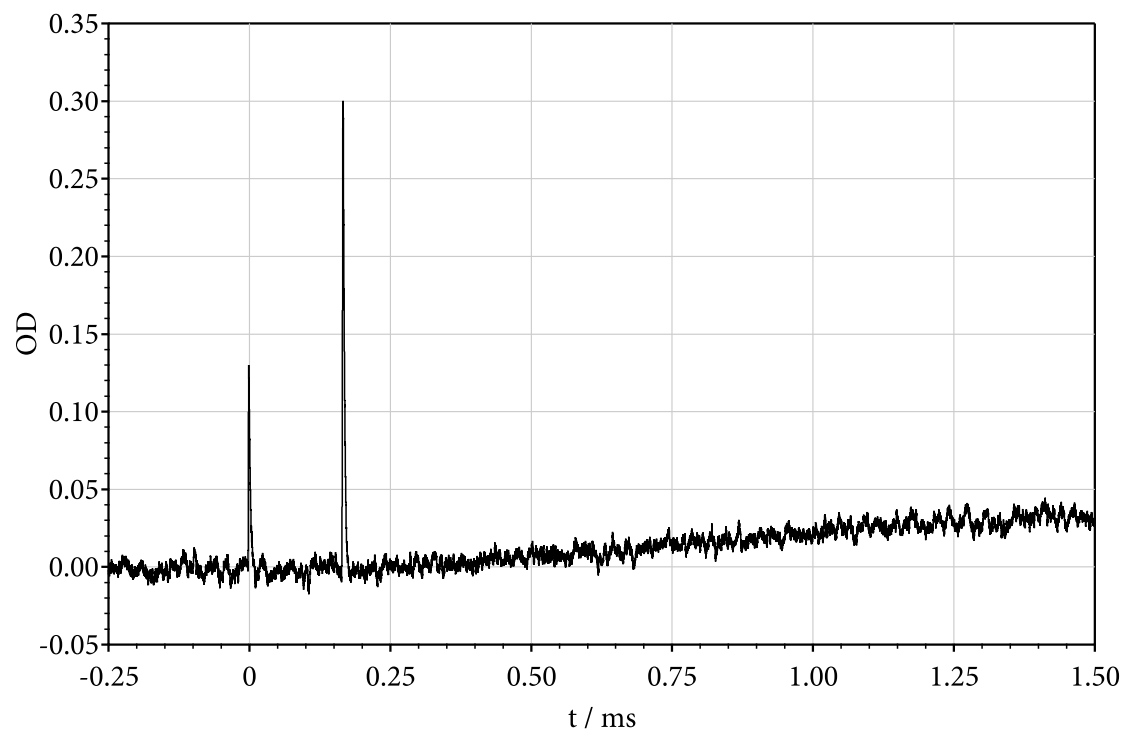

Abb. 3.3: Zerfall von $\mathrm{C}_{2} \mathrm{HF}_{5}: \lambda=248 \mathrm{~nm} \mathrm{~T}=1269 \mathrm{~K},[\mathrm{Ar}]=8.98 \cdot 10^{-5} \mathrm{~mol} / \mathrm{cm}^{3}$

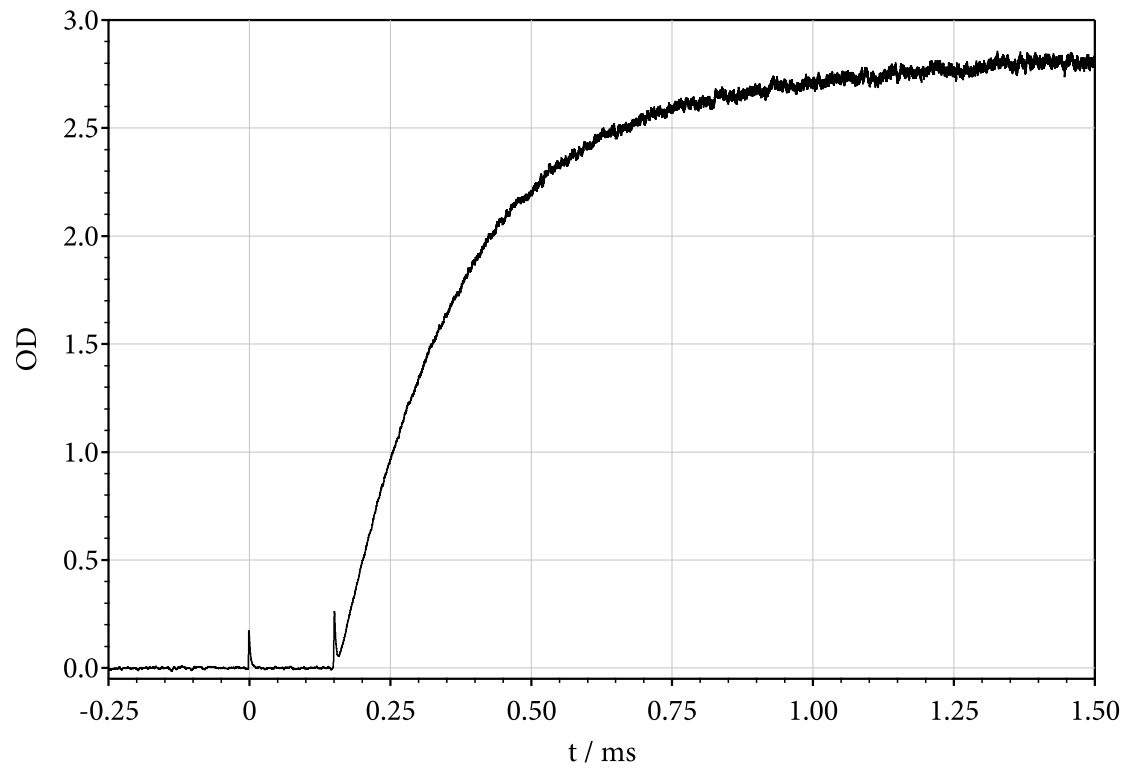

Abb. 3.4: Zerfall von $\mathrm{C}_{2} \mathrm{HF}_{5}: \lambda=248 \mathrm{~nm} \mathrm{~T}=1596 \mathrm{~K},[\mathrm{Ar}]=7.06 \cdot 10^{-5} \mathrm{~mol} / \mathrm{cm}^{3}$ 


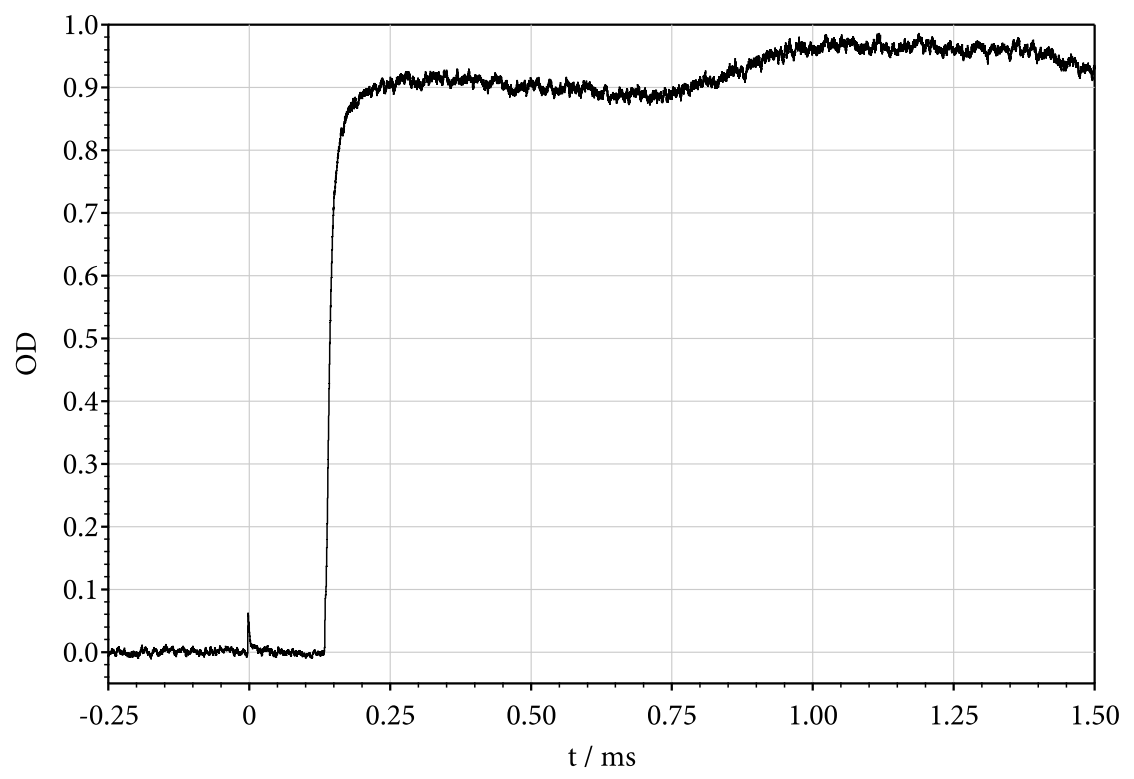

Abb. 3.5: Zerfall von $\mathrm{C}_{2} \mathrm{HF}_{5}: \lambda=248 \mathrm{~nm} \mathrm{~T}=2049 \mathrm{~K},[\mathrm{Ar}]=1.98 \cdot 10^{-5} \mathrm{~mol} / \mathrm{cm}^{3}$

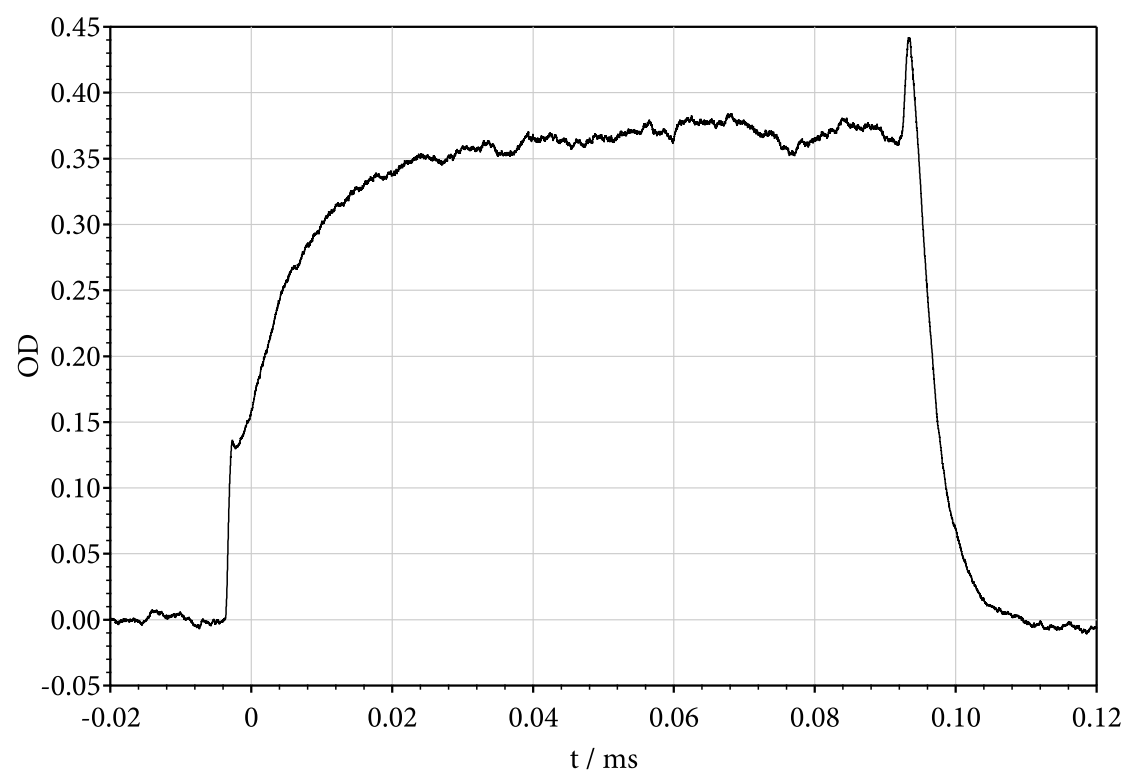

Abb. 3.6: Zerfall von $\mathrm{C}_{2} \mathrm{HF}_{5}: \lambda=248 \mathrm{~nm} \mathrm{~T}=2017 \mathrm{~K},[\mathrm{Ar}]=7.02 \cdot 10^{-6} \mathrm{~mol} / \mathrm{cm}^{3}$ 


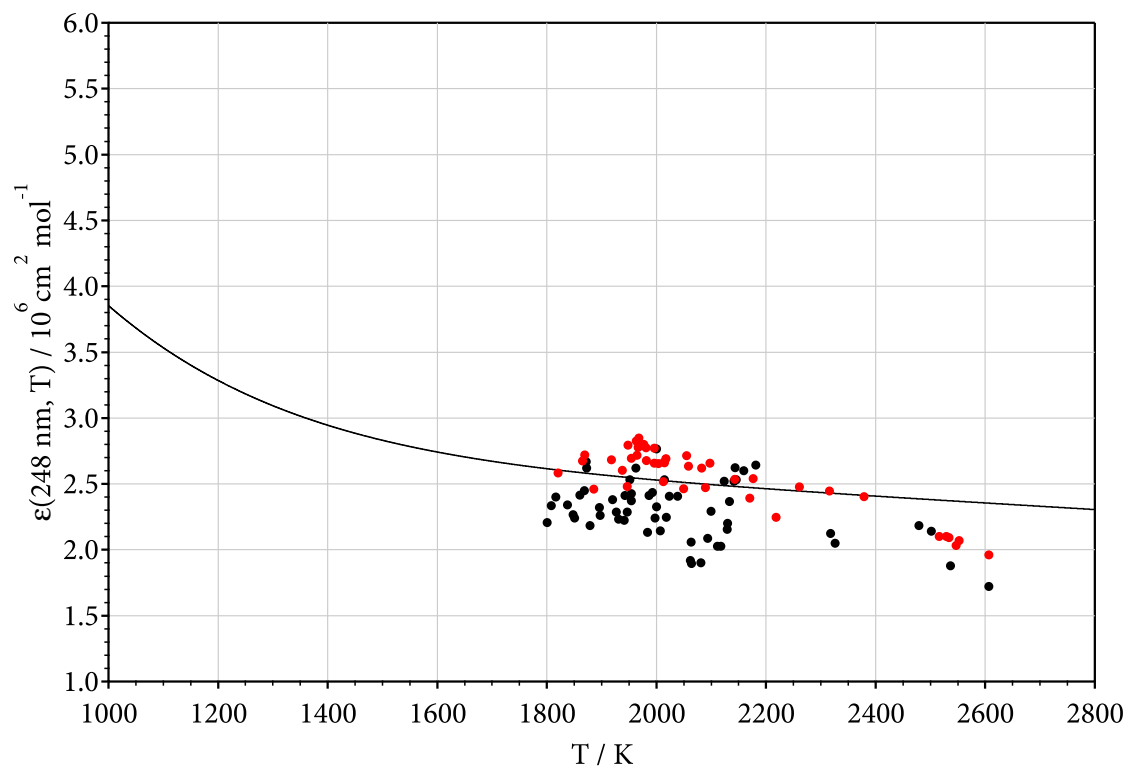

Abb. 3.7: Absorptionskoeffizienten von $\mathrm{C}_{2} \mathrm{HF}_{5}$ und $\mathrm{C}_{3} \mathrm{HF}_{7}$ im Vergleich zur Gaussanpassung von Gl. (3.2), $\bullet: \mathrm{C}_{2} \mathrm{HF}_{5}, \bullet: \mathrm{C}_{3} \mathrm{HF}_{7}$

ist allerdings temperaturabhängig, d.h. bei kleinen Temperaturen ist während der zugänglichen Messzeit kein voller Umsatz zu erhalten. Die Grafik 3.7 illustriert die aus dem $\mathrm{OD}_{\text {max }}$ erhaltenen Absorptionskoeffizienten für $\mathrm{C}_{2} \mathrm{HF}_{5}$ und $\mathrm{C}_{3} \mathrm{HF}_{7}$. Hierbei wurden die Faktoren ( 2 für $\mathrm{C}_{2} \mathrm{HF}_{5}$ und 3 für $\mathrm{C}_{3} \mathrm{HF}_{7}$ ) bereits mit einbezogen. Es zeigt sich dann eine gute Übereinstimmung mit der in 3.1.1.1 beschriebenen GaussAnpassung an den Absorptionskoeffizienten $\epsilon$.

\subsubsection{Visuelle Bewertung der Messdaten}

Als nächster Schritt bietet sich an, die gemessenen Absorptions-Zeit-Profile rein visuell zu beurteilen. Unter der Annahme der vollständigen Konversion von $\mathrm{C}_{2} \mathrm{HF}_{5}$ bzw. $\mathrm{C}_{3} \mathrm{HF}_{7} \mathrm{zu} \mathrm{CF}_{2}$, ist ein Verlauf nach einem Geschwindigkeitsgesetz 1. Ordnung zu erwarten (Abb. 3.5). Es zeigt sich jedoch, dass die Auftragungen häufig einen anderen Verlauf zeigen. Während der vordere Teil nach einem Geschwindigkeitsgesetz 1. Ordnung zu reagieren scheint, ergeben sich danach größere Abweichungen von diesem Verhalten . Bei kleinen Konzentrationen und niedrigen Temperaturen wird nach einer Induktionszeit eine Beschleunigung der Reaktion sichtbar. Bei hohen 


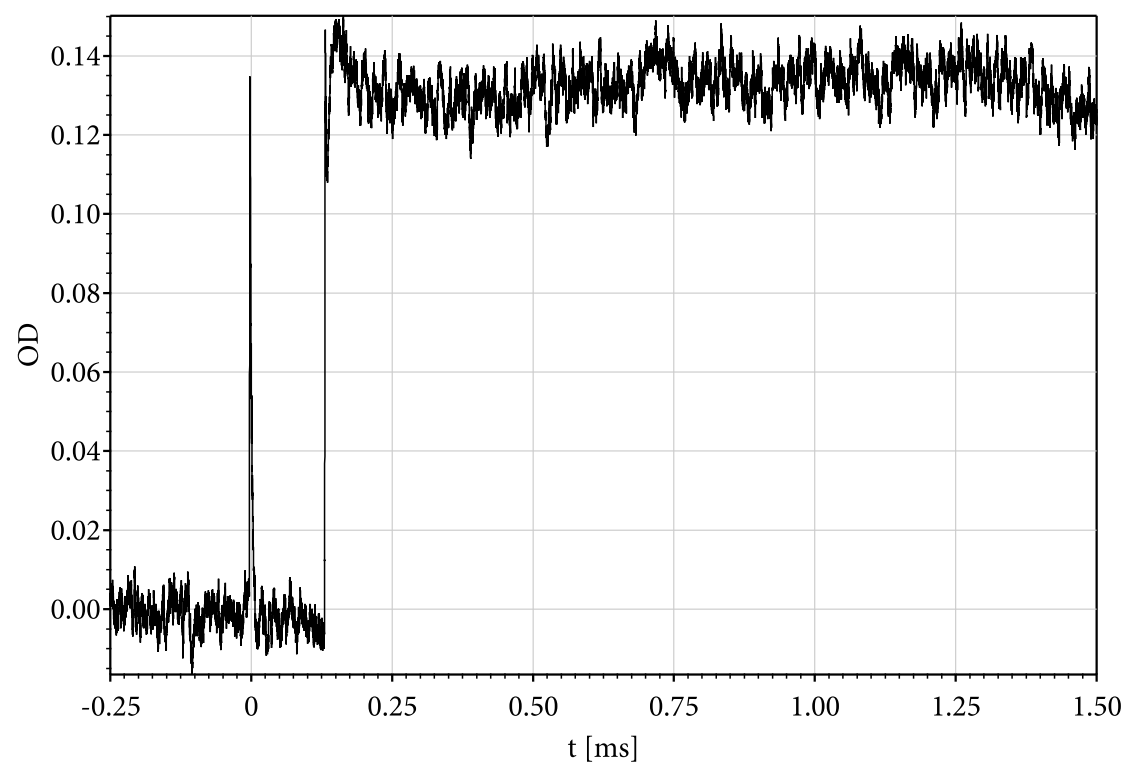

Abb. 3.8: Zerfall von $\mathrm{C}_{2} \mathrm{HF}_{5}$ : $\mathrm{T}=2065 \mathrm{~K}, 67 \mathrm{ppm}$.

Temperaturen ist ein $\mathrm{CF}_{2}$-Verbrauch zu beobachten, nachdem die primäre Bildung von $\mathrm{CF}_{2}$ durch Dissoziation beendet war (siehe Abb. 3.8.

\subsubsection{Bestimmung der Geschwindigkeitskonstanten}

Als folgender Teil der Auswertung wird eine Geschwindigkeitskonstante erster Ordnung $k$ bestimmt.

Jede Messung folgt, zumindest im Bereich der Anfangssteigung, einem Geschwindigkeitsgesetz pseudo-erster Ordnung, da die Argonkonzentration bei jedem Mischverhältnis um ein vielfaches größer ist, als die Reaktandenkonzentration. Während die Zerfallsreaktion im Niederdruckbereich sowohl von der Reaktanden, als auch von der Badgaskonzentration abhängig ist, kommt es im Hochdruckbereich nur noch zu einer Abhängigkeit von der Reaktandenkonzentration. Die Argonkonzentration hat dann keinen Einfluss mehr auf die Geschwindigkeit der Reaktion. Im Falloffbereich liegt die Reaktionsordnung zwischen einer Reaktion erster und nullter Ordnung bezüglich der Argonkonzentration.

Die Bildung von $\mathrm{CF}_{2}$ verläuft nach einem Geschwindigkeitsgesetz 1. Ordnung: 


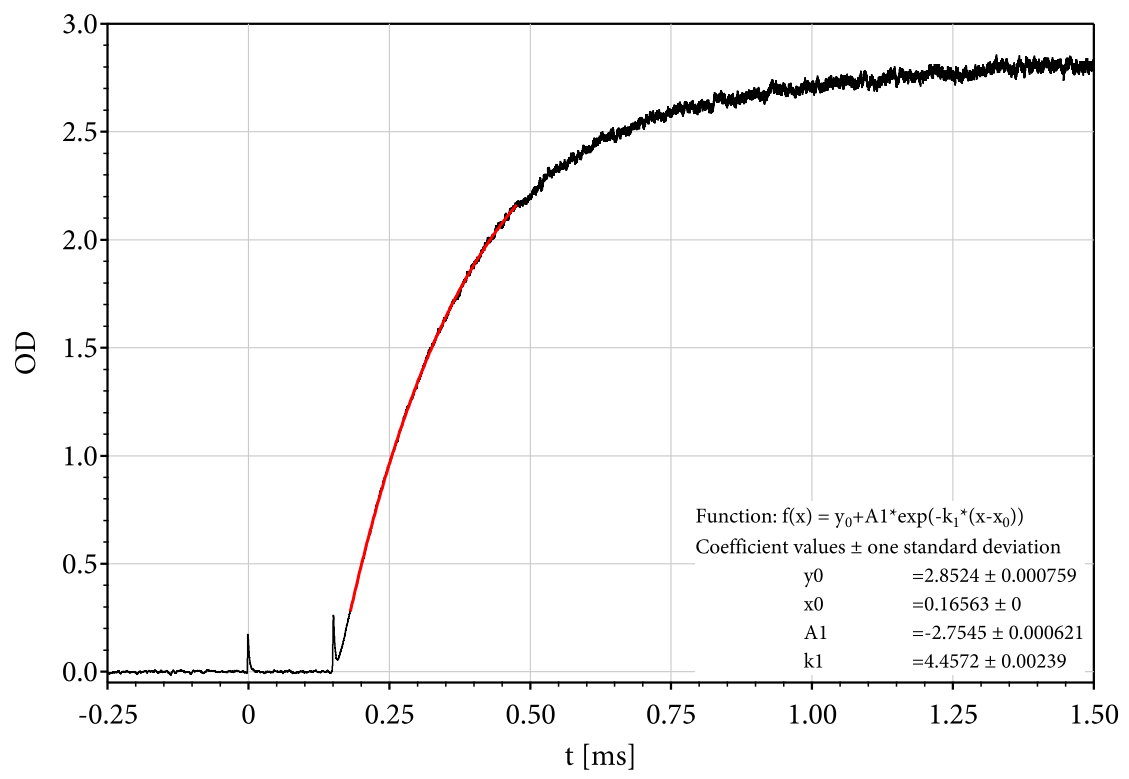

Abb. 3.9: Bestimmung der Geschwindigkeitskonstanten aus der Anfangssteigung

$$
\left[\mathrm{CF}_{2}\right]=\left[\mathrm{CF}_{2}\right]_{t=\infty} \cdot\left[1-\exp \left(k_{a} t\right)\right]
$$

wobei

$$
\left[\mathrm{CF}_{2}\right]_{\mathrm{t}=\infty}=2\left[\mathrm{C}_{2} \mathrm{HF}_{5}\right]_{\mathrm{t}=0}
$$

entspricht.

Zur Bestimmung der Geschwindigkeitskonstante wurde mit einer einfach-exponentiellen Funktion die Anfangssteigung der Absorbanz angepasst. Wichtig ist dabei, erst nach dem Schlierensignal mit der Anpassung zu beginnen und weiterhin ausschließlich den Anfangsbereich der Steigung zu erfassen, um sicherzugehen, dass die $\mathrm{CF}_{2}$-Bildung dort nach einem Geschwindigkeitsgesetz 1. Ordnung verläuft. Die sich daraus ergebene Steigung wird dann genutzt, um die Geschwindigkeitskonstanten zu berechnen. 


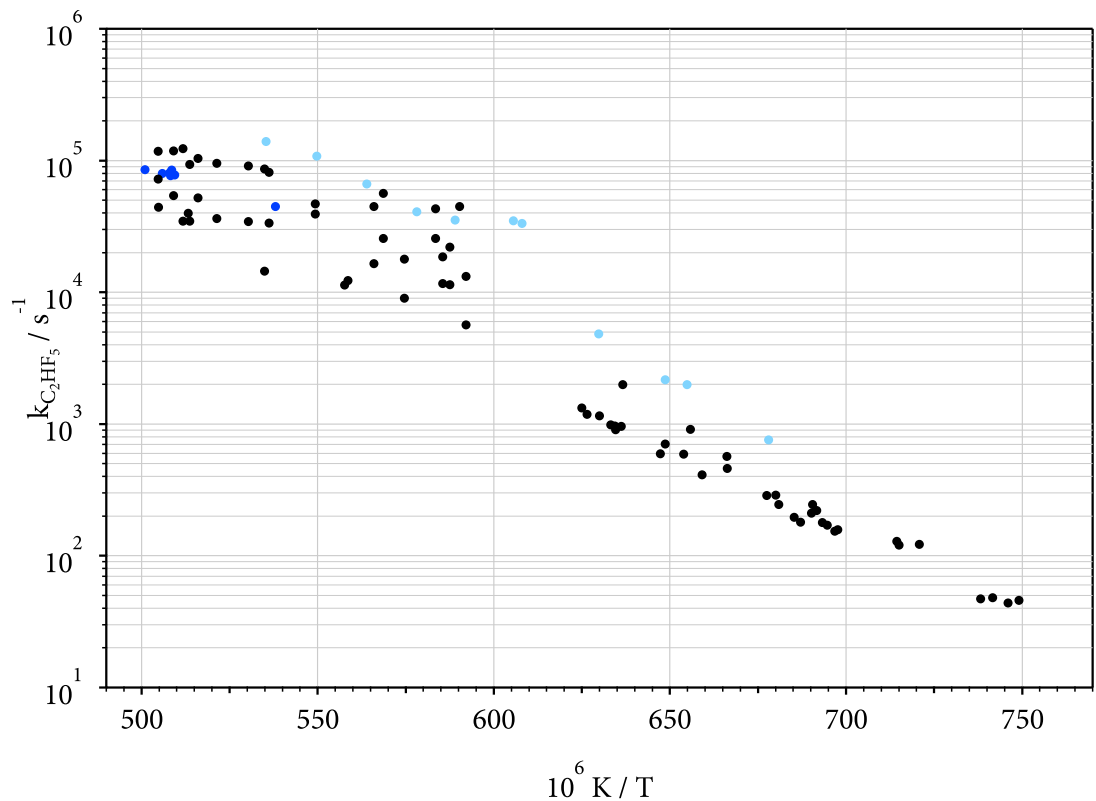

Abb. 3.10: Arrhenius-Auftragung der Geschwindigkeitskonstante von $\mathrm{C}_{2} \mathrm{HF}_{5}: \bullet: 1000 \mathrm{ppm}, \bullet$ : $500 \mathrm{ppm}, \circ: 70 \mathrm{ppm}$

Die aus den jeweiligen Einzelmessungen erhaltenen Geschwindigkeitskonstanten werden logarithmisch gegen das Inverse der Temperatur aufgetragen (siehe Abb. 3.10 für $\mathrm{C}_{2} \mathrm{HF}_{5}$ und $\mathrm{Abb}$. 3.11 für $\mathrm{C}_{3} \mathrm{HF}_{7}$ ), es wird also eine Arrhenius-Auftragung gewählt. Bei Betrachtung der Geschwindigkeitskonstanten wird deutlich, dass die berechneten $k$ eine Abhängigkeit von der Konzentration zeigen. Deshalb ist es nicht sinnvoll eine Gesamtgeschwindigkeitskonstante anzugeben. Im Folgenden sind daher die Geschwindigkeitskonstanten von $\mathrm{C}_{2} \mathrm{HF}_{5} k_{\mathrm{C}_{2} \mathrm{HF}_{5}, l}$ (für niedrige Konzentrationen um $70 \mathrm{ppm}$ ) und $k_{\mathrm{C}_{2} \mathrm{HF}_{5}, h}$ (für hohe Konzentrationen um $1000 \mathrm{ppm}$ ), sowie die Geschwindigkeitskonstanten von $\mathrm{C}_{3} \mathrm{HF}_{7} k_{\mathrm{C}_{3} \mathrm{HF}_{7}, l}$ (für niedrige Konzentrationen um $70 \mathrm{ppm}$ ) und $k_{\mathrm{C}_{3} \mathrm{HF}_{7}, h}$ (für hohe Konzentrationen um $1000 \mathrm{ppm}$ ) angegeben:

$$
\begin{aligned}
& k_{\mathrm{C}_{2} \mathrm{HF}_{5}, l}=8.2 \cdot 10^{14} \cdot \exp (-41400 \mathrm{~K} / T) \mathrm{s}^{-1} \\
& k_{\mathrm{C}_{3} \mathrm{HF}_{7}, l}=4.2 \cdot 10^{14} \cdot \exp (-36750 \mathrm{~K} / T) \mathrm{s}^{-1}
\end{aligned}
$$




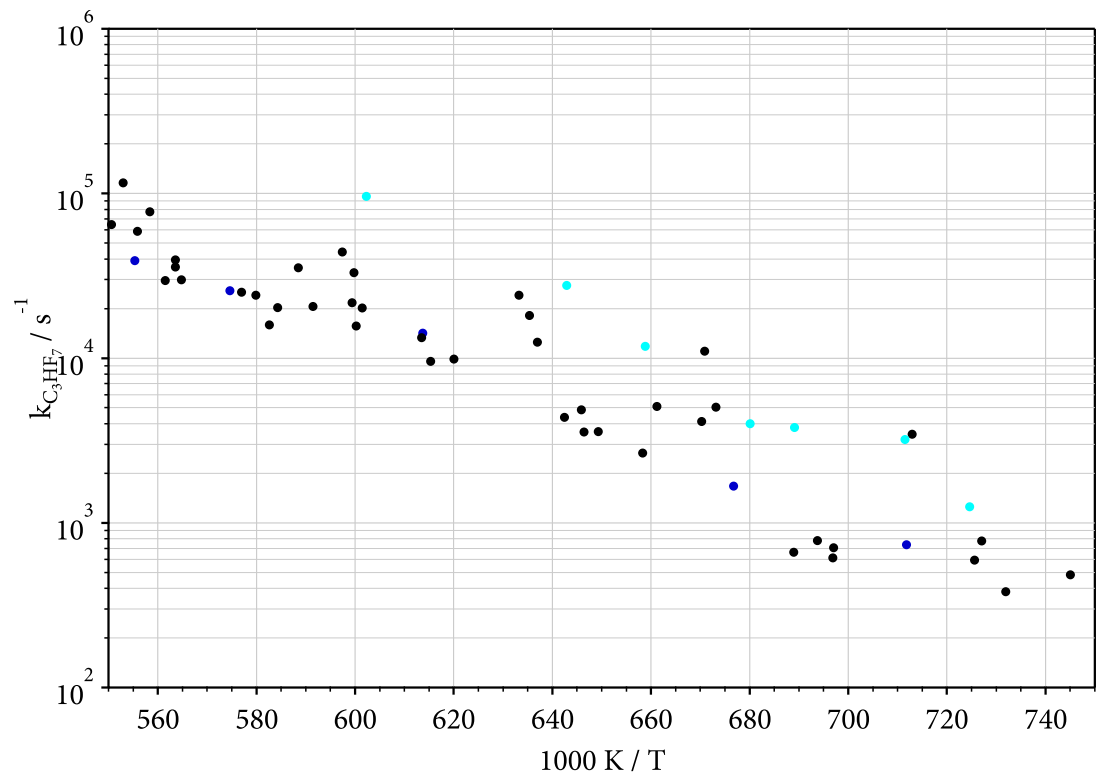

Abb. 3.11: Arrhenius-Auftragung der Geschwindigkeitskonstante von $\mathrm{C}_{3} \mathrm{HF}_{7}: \bullet: 1000 \mathrm{ppm}, \bullet$ : $600 \mathrm{ppm}, \circ: 70 \mathrm{ppm}$

$$
\begin{aligned}
& k_{\mathrm{C}_{2} \mathrm{HF}_{5}, h}=1.2 \cdot 10^{13} \cdot \exp (-36580 \mathrm{~K} / T) \mathrm{s}^{-1} \\
& k_{\mathrm{C}_{3} \mathrm{HF}_{7}, h}=5.7 \cdot 10^{13} \cdot \exp (-25440 \mathrm{~K} / T) \mathrm{s}^{-1}
\end{aligned}
$$

Diese Geschwindigkeitskonstanten sind zusammengesetzt und können Effekte von sekundären Reaktionen beinhalten, sowie auch durch den Druck beeinflusst sein. 


\section{Kapitel 4}

\section{Diskussion}

\subsection{Resultate zur Pyrolyse des Pentafluorethans}

Nachdem alle Schritte der Auswertung vorgenommen wurden, können die Resultate beurteilt werden. Auf den ersten Blick könnte man aus den Messungen schließen, dass die Reaktion nach einem Geschwindigkeitsgesetz 1. Ordnung verläuft. Dies würde durch die Ausbeutenbestimmung unterstützt.

Es könnte angenommen werden, dass die Reaktion über zwei Stufen verläuft und dabei pro Molekül $\mathrm{C}_{2} \mathrm{HF}_{5}$ zwei $\mathrm{CF}_{2}$ gebildet werden. Im Anschluss an Reaktion (1.1) würde hierbei die folgende Reaktion:

$$
\mathrm{C}_{2} \mathrm{~F}_{4}+(\mathrm{M}) \longrightarrow 2 \mathrm{CF}_{2}+(\mathrm{M})
$$

auftreten.

Bei näherer Betrachtung der aufgetragenen Messungen wird jedoch deutlich, dass größere Abweichungen zu diesem Mechanismus vorliegen. Induktionszeiten bzw. eine verzögerte Bildung von $\mathrm{CF}_{2}$ deuten darauf hin, dass die Reaktion nicht nach o.g. Schema verläuft. Dies legt vor allem die Beobachtung einer Konzentrationsabhängigkeit der effektiven Geschwindigkeitkonstanten in Abb. 3.10 nahe.

Ein direkter Vergleich von Messungen bei 70 ppm und 500-1000 ppm bei gleicher Temperatur liefert ein aufschlussreiches Bild. Die Abbildungen 4.1 und 4.2 zeigen, dass bei unveränderter Ausbeute die $\mathrm{CF}_{2}$-Bildung unterschiedlich verläuft und von Gleichung 3.4 abweicht.

Dies bestätigt sich in der Auftragung Abb. 3.10. Dabei wird sichtbar, dass es einen Übergang zwischen den effektiven Geschwindigkeitskonstanten für hohe und niedrige Konzentrationen gibt, wie durch die Gleichungen (3.6) und (3.8) beschrieben wird. 


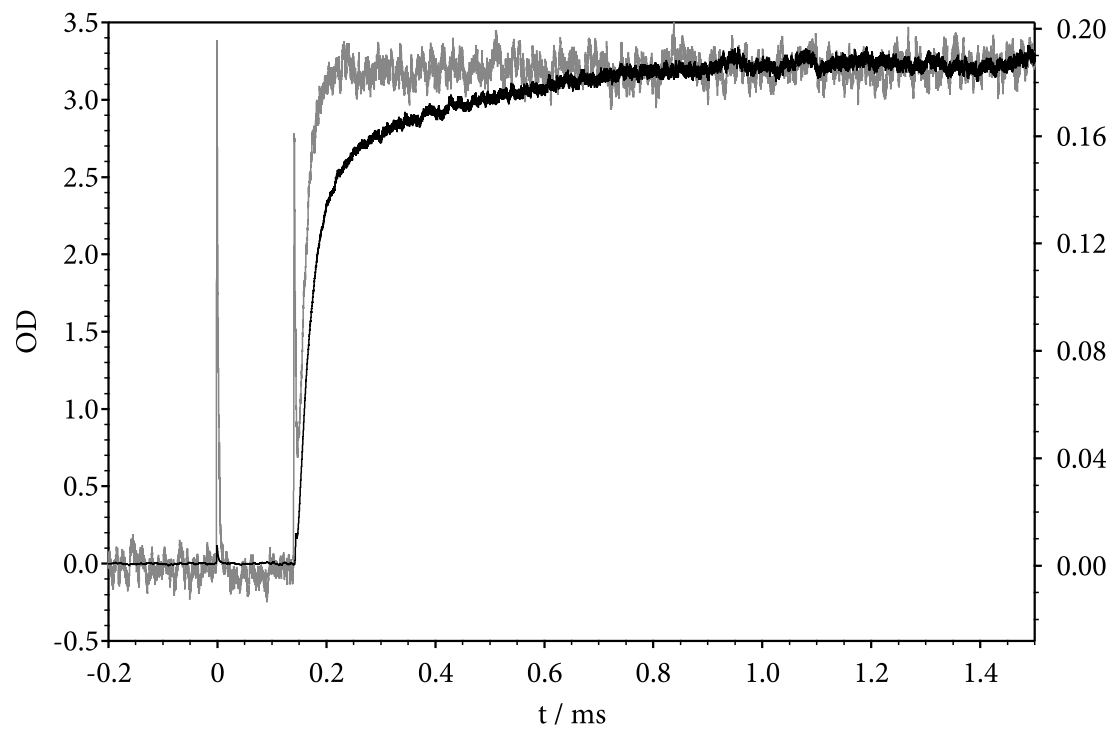

Abb. 4.1: Vergleich der $\mathrm{CF}_{2}$-Bildung aus $\mathrm{C}_{2} \mathrm{HF}_{5}$ bei niedrigen (grau, $70 \mathrm{ppm}$ ) und hohen Konzentrationen (schwarz, 1000 ppm) bei etwa $1800 \mathrm{~K}$

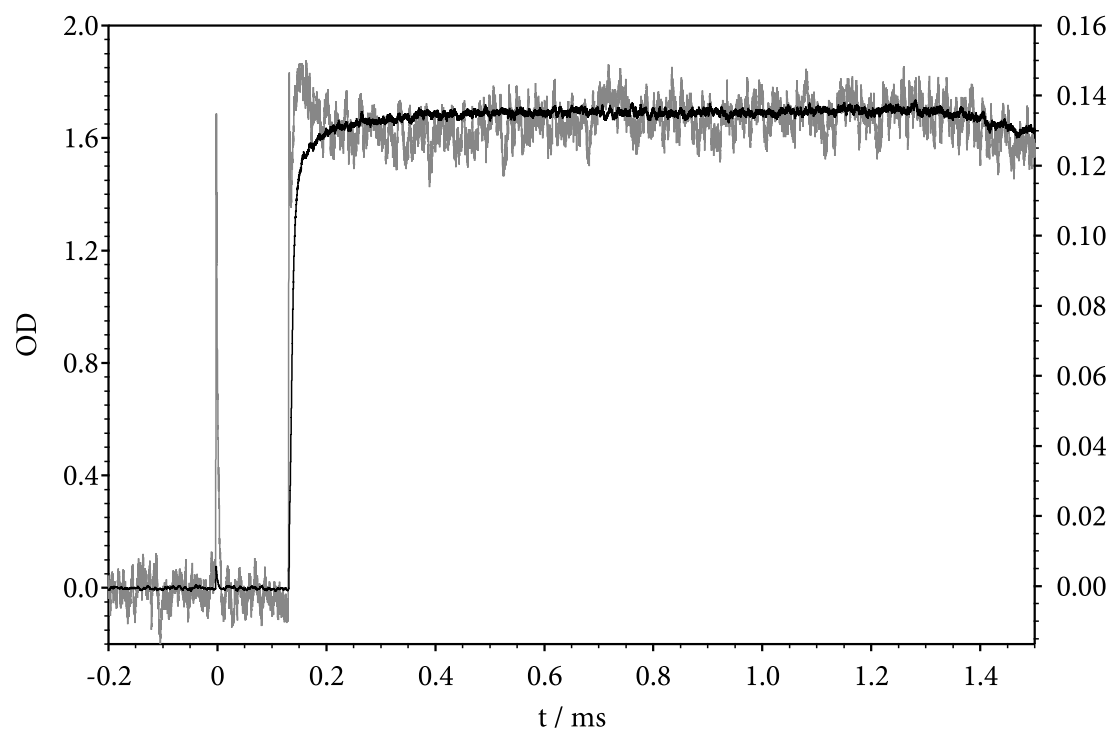

Abb. 4.2: Vergleich der $\mathrm{CF}_{2}$-Bildung aus $\mathrm{C}_{2} \mathrm{HF}_{5}$ bei niedrigen (grau, $70 \mathrm{ppm}$ ) und hohen Konzentrationen (schwarz, $600 \mathrm{ppm}$ ) bei etwa $2000 \mathrm{~K}$ 


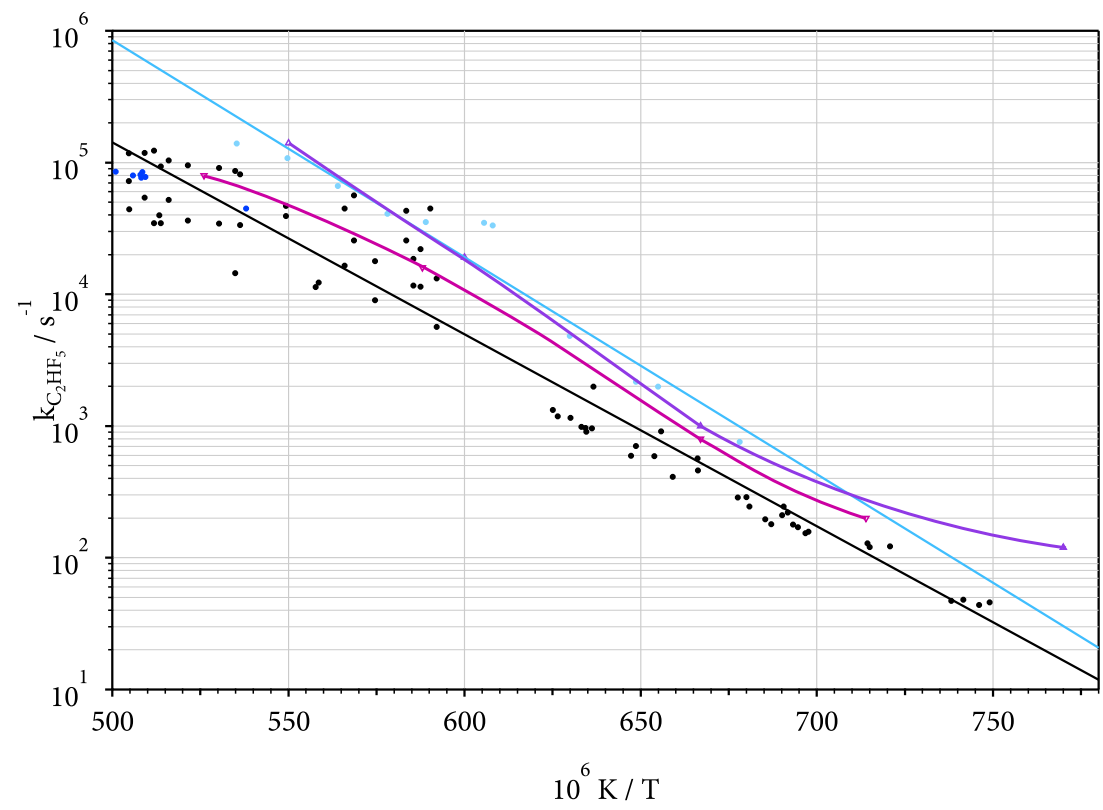

Abb. 4.3: Arrheniusauftragung der Geschwindigkeitskonstante von $\mathrm{C}_{2} \mathrm{HF}_{5}$ : : $70 \mathrm{ppm}$; •: 1000 ppm; 600 ppm; blaue Linie: $k_{\mathrm{C}_{2} \mathrm{H}_{5}, l}$ (Gl. 3.6), schwarze Linie: $k_{\mathrm{C}_{2} \mathrm{HF}_{5}, h}$ (Gl. 3.8), violette Linie: $\left.k_{\mathrm{C}_{2} \mathrm{~F}_{6}} \mid 28\right]$; magenta Linie: $\left.k_{\mathrm{CF}_{3} \mathrm{H}}|19|\right)$

Die Verringerung der Geschwindigkeitskonstanten 1. Ordnung von niedriger zu hoher Konzentration ist signifikant. Abbildung 4.3 zeigt die bereits bekannte ArrheniusAuftragung, in die nun jedoch Geschwindigkeitskonstanten der Bindungsbruchreaktion

$$
\mathrm{C}_{2} \mathrm{~F}_{6} \longrightarrow 2 \mathrm{CF}_{3}
$$

aus 28 sowie Geschwindigkeitskonstanten für die $\mathrm{CF}_{2}$-Bildung durch die Reaktion:

$$
\mathrm{CF}_{3} \mathrm{H} \longrightarrow \mathrm{CF}_{2}+\mathrm{HF}
$$

bei gleicher Argonkonzentration $\left([\mathrm{Ar}]=8 \cdot 10^{-5} \mathrm{~mol} \mathrm{~cm}^{-3}\right)$, aus $\mid 19$ einbezogen wurden. 
Hierbei zeigt sich, dass die Geschwindigkeitskonstanten von Reaktion (4.2) nahe denen für den $\mathrm{C}_{2} \mathrm{HF}_{5}$-Zerfalls bei geringer Konzentration liegen, während die Geschwindigkeitskonstanten von Reaktion (4.3) eher denen hoher Konzentration zuzuordnen sind.

\subsubsection{Modellierung der primären Reaktionsschritte}

Da das während des $\mathrm{C}_{2} \mathrm{HF}_{5}$-Zerfalls gebildete $\mathrm{CF}_{2}$ nicht aus dem ersten Reaktionsschritt stammt, erscheint es notwendig, die primären Reaktionswege quantenchemisch zu untersuchen. In der Literatur [11, 12, 29] finden sich einige, jedoch unvollständige, quantenchemische Rechnungen hierzu, so dass weitere Berechnungen durchzuführen sind.

Zur Beurteilung der thermochemischen Eigenschaften von Übergangszuständen werden von Carlos Cobos für eine Auswahl möglicher Zerfallskanäle Dichtefunktionaltheorie (DFT) und zusammengesetzte Berechnungen mit der ab initio Methode vorgenommen. Zuerst werden zwei DFT-Methoden (BMK [30] und M06-2X [31]) mit dem 6-311++G(3df,2pd) Basissatz kombiniert. Danach folgt eine ab initio Rechnung mittels der CBS-QBS (Complete Basis Set) Methode [32], sowie G3MP2B3 [33] und G4 [34 Gaussian-Modell-Rechnungen mit dem Gaussian 09 Algorithmus [35]. Folgende primäre Reaktionsschritte werden berücksichtigt:

$$
\begin{aligned}
& \mathrm{CF}_{3} \mathrm{CF}_{2} \mathrm{H} \longrightarrow \mathrm{CF}_{3} \mathrm{CF}+\mathrm{HF} \\
& \mathrm{CF}_{3} \mathrm{CF}_{2} \mathrm{H} \longrightarrow \mathrm{CF}_{2} \mathrm{CF}_{2}+\mathrm{HF} \\
& \mathrm{CF}_{3} \mathrm{CF}_{2} \mathrm{H} \longrightarrow \mathrm{CF}_{4}+\mathrm{CHF} \\
& \mathrm{CF}_{3} \mathrm{CF}_{2} \mathrm{H} \longrightarrow \mathrm{CF}_{3} \mathrm{H}+\mathrm{CF}_{2} \\
& \mathrm{CF}_{3} \mathrm{CF}_{2} \mathrm{H} \longrightarrow \mathrm{CF}_{3}+\mathrm{CF}_{2} \mathrm{H}
\end{aligned}
$$




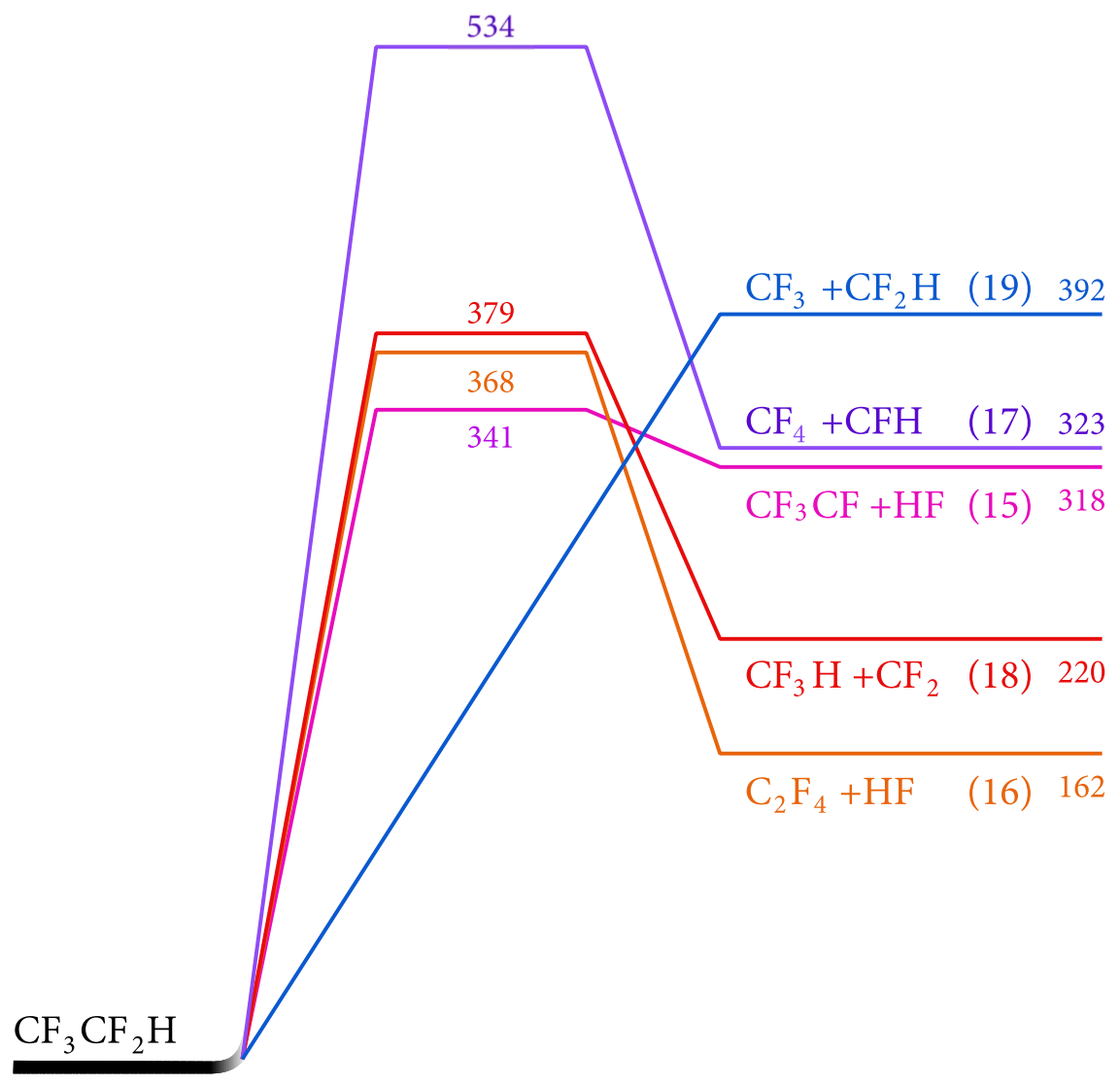

Abb. 4.4: Energieprofil der Reaktionen von $\mathrm{C}_{2} \mathrm{HF}_{5}$ 
Tab. 4.1: Reakionsenthalpien für die Zerfallskanäle von $\mathrm{C}_{2} \mathrm{HF}_{5}$ bei $0 \mathrm{~K}$ in kJ mol${ }^{-1}$

\begin{tabular}{|c|c|c|c|c|c|c|}
\hline Reaktionsprodukt & $\begin{array}{r}\text { BMK } \\
30\end{array}$ & $\begin{array}{c}\text { MO6-2X } \\
\text { 31 }\end{array}$ & $\begin{array}{c}\text { CBS-QB3 } \\
\mid 32\end{array}$ & $\begin{array}{c}\text { G3MP2B3 } \\
33\end{array}$ & $\begin{array}{l}\mathrm{G} 4 \\
34\end{array}$ & $\begin{array}{r}\text { Table } \\
36\end{array}$ \\
\hline $\mathrm{CF}_{3} \mathrm{CF}+\mathrm{HF}(4.4)$ & 333 & 337 & 326 & 324 & 318 & - \\
\hline $\mathrm{CF}_{2} \mathrm{CF}_{2}+\mathrm{HF}(4.5)$ & 170 & 172 & 164 & 164 & 162 & 166 \\
\hline $\mathrm{CF}_{4}+\mathrm{CFH}(4.6)$ & 331 & 332 & 330 & 329 & 323 & 332 \\
\hline $\mathrm{CF}_{3} \mathrm{H}+\mathrm{CF}_{2}(4.7)$ & 220 & 230 & 226 & 226 & 220 & 232 \\
\hline $\mathrm{CF}_{3}+\mathrm{CF}_{2} \mathrm{H}(4.8)$ & 389 & 397 & 406 & 405 & 392 & 410 \\
\hline
\end{tabular}

Tab. 4.2: Barrierenhöhen für die Zerfallskanäle von $\mathrm{C}_{2} \mathrm{HF}_{5}$ bei $0 \mathrm{~K}$ in kJ mol${ }^{-1}$

\begin{tabular}{l|ccccc}
\hline Reaktionsprodukt & BMK & MO6-2X & CBS-QB3 & G3MP2B3 & G4 \\
& $\mid 30$ & $\mid 31$ & 32 & 33 & 34 \\
\hline $\mathrm{CF}_{3} \mathrm{CF}+\mathrm{HF}(4.4)$ & 352 & 355 & 348 & 341 & 341 \\
$\mathrm{CF}_{2} \mathrm{CF}_{2}+\mathrm{HF}(4.5)$ & 364 & 369 & 373 & 367 & 368 \\
$\mathrm{CF}_{4}+\mathrm{CFH}(4.6)$ & 544 & 536 & 538 & 532 & 534 \\
$\mathrm{CF}_{3} \mathrm{H}+\mathrm{CF}_{2}(4.7)$ & 396 & 400 & 381 & 379 & 379 \\
$\mathrm{CF}_{3}+\mathrm{CF}_{2} \mathrm{H}(4.8)$ & 389 & 397 & 406 & 405 & 392 \\
\hline
\end{tabular}

Bei genauerer Betrachtung zeigen Tabelle 4.2 und Abbildung 4.4 , dass drei Eliminationsprozesse existieren, welche direkt oder mit einem zweiten, sehr schnellen Prozess, als Folge $\mathrm{zu} \mathrm{CF}_{2}$ führen. Die elektronische Barriere für die Isomerisierung

$$
\mathrm{CF}_{3} \mathrm{CF} \longrightarrow \mathrm{C}_{2} \mathrm{~F}_{4}
$$

wird mit dem G3MP2B3 Verfahren zu $148 \mathrm{~kJ} \mathrm{~mol}^{-1}$ berechnet, d.h. diese Reaktion verläuft sehr schnell. Andererseits ist die Sekundärdissoziation von $\mathrm{C}_{2} \mathrm{~F}_{4}$ mit einer Bindungsenergie von $\Delta H_{0}^{0}=282 \mathrm{~kJ} \mathrm{~mol}^{-1}$ schnell genug |13], so dass die $\mathrm{CF}_{2}$-Bildung der Reaktionen (4.4) und (4.5) durch die Geschwindigkeit der Folgeprozesse dominiert wird. Die drei Eliminiationreaktionen (4.4), (4.5) und (4.6) werden durch starre aktivierte Komplexe beschrieben. Sie konkurrieren mit dem lockeren aktivierten Komplex der C-C-Bindungsbruchreaktion (4.8). In [37] wurde mittels InfrarotMultiphotonen-Dissoziation bereits eine Dominanz von Reaktion (4.8) über (4.4) gefunden. In den Arbeiten von [12] und [9| wurde ein solches Verhalten allerdings nur für Temperaturen über $1350 \mathrm{~K}$ vorgeschlagen, während das Gegenteil für niedrige Temperaturen beobachtet wurde. 
Um den offenbar vorhandenen Übergang zwischen der Reaktion (4.4) mit starrem aktivierten Komplex bei niedrigen Temperaturen und Reaktion (4.8) mit locker aktiviertem Komplex bei hohen Temperaturen besser verstehen zu können, werden auch die Geschwindigkeitskonstanten der beiden Reaktionen modelliert. Die dafür benötigten Frequenzen der aktivierten Komplexe, sowie die Frequenzen der Muttermoleküle, werden mittels DFT-Berechnungen bestimmt (siehe Anhang). Als Resultat für Reaktion (4.4) ergibt sich eine Hochdruckgeschwindigkeitskonstante:

$$
k_{4.4, \infty}=5.5 \cdot 10^{14} \cdot \exp \left(-365 \mathrm{~kJ} \mathrm{~mol}^{-1} / R T\right) \mathrm{s}^{-1}
$$

Da die vorliegenden Experimente und frühere Arbeiten [9 11, 12] eine extrem große Druckdifferenz aufweisen $\left(10^{-1}-10^{4}\right.$ Torr) wird außerdem die Niederdruckgeschwindigkeitskonstante $k_{4.4,0}[38-[40]$ sowie der Verbreiterungsfaktor der Falloff-Kurve $F_{c e n t, 4.4}$ bestimmt. Obwohl die Falloff-Kurve sehr breit ist $\left(F_{c e n t, 4.4}<0.1\right.$, bei $\mathrm{T}>$ $1000 \mathrm{~K}$ ) liegt Reaktion (4.4) in dieser Arbeit im Gegensatz zu Reaktion (4.3) nahe des Hochdrucklimits. Hierbei ist zu beachten, dass die modellierte Geschwindigkeitskonstante $k_{4.4}$ näher an den Ergebnissen von [11] $\left(k_{4.4, \infty}(1300 \mathrm{~K})=2.7 \mathrm{~s}^{-1}\right.$ und $\left.k_{4.4}(1300 \mathrm{~K})=7.6 \mathrm{~s}^{-1}\right)$ liegt, als an der Geschwindigkeitskonstante von [9] $\left(k_{4.4}(1300 \mathrm{~K})=37 \mathrm{~s}^{-1}\right)$. Diese Diskrepanz ist vermutlich auf die unterschiedlichen Reaktandenkonzentrationen zurückzuführen. Auf ähnliche Weise werden die Hochdruckgeschwindigkeitskonstanten für die Reaktionen (4.5) und (4.7) modelliert. Auf Reaktion (4.6) wird nicht näher eingegangen, da die Energiebarriere zu hoch ist, um zur Reaktion beizutragen (siehe Tabelle 4.2. Man erhält folgende Geschwindigkeitskonstanten für die Reaktionen (4.5) und (4.7):

$$
\begin{aligned}
& k_{4.5, \infty}=2.1 \cdot 10^{14} \cdot \exp \left(-389 \mathrm{~kJ} \mathrm{~mol}^{-1} / R T\right) \mathrm{s}^{-1} \\
& k_{4.7, \infty}=1.6 \cdot 10^{15} \cdot \exp \left(-399 \mathrm{~kJ} \mathrm{~mol}^{-1} / R T\right) \mathrm{s}^{-1}
\end{aligned}
$$

Daraus ergibt sich direkt, dass diese beiden Reaktionskanäle gegenüber Reaktion (4.4) zu vernachlässigen sind.

Vergleicht man nun das modellierte $k_{4.4, \infty}$ mit dem gemessenen $k_{\mathrm{C}_{2} \mathrm{HF}_{5}, l}$ aus Gleichung (3.6) und betrachtet man die Konzentrationsabhängigkeit der Geschwindigkeitskonstanten, liegt der Schluss nahe, dass nicht die HF-Eliminination (4.4), sondern der C-C-Bindungsbruch (4.8) die $\mathrm{C}_{2} \mathrm{HF}_{5}$-Zersetzung dominiert (bei den 
hier genutzten Bedingungen und Temperaturen über $1400 \mathrm{~K}$ ). Die Experimente zur Multiphotonen-Dissoziation aus den Arbeiten von [41] und [42] unterstützen dieses Ergebnis.

Energieabhängige spezifische Geschwindigkeitskonstanten $k(E)$ für diesen Prozess werden in 29$]$ mit denen für Reaktion (4.4) verglichen. Diese Rechnungen sind allerdings nur vorläufig. Da das detaillierte Wissen über die Potentialhyperfläche für den Bindungsbruch von $\mathrm{CF}_{3}-\mathrm{CF}_{2} \mathrm{H}$ fehlt, um solche Berechnungen selbst durchzuführen, wird sich im Folgenden auf den Vergleich mit dem Bindungsbruch von $\mathrm{CF}_{3}-\mathrm{CF}_{3}$ aus der Arbeit von [20] bezogen. Auf Basis von druck- und temperaturabhängigen Experimenten für die Reaktion [20

$$
\mathrm{CF}_{3} \mathrm{CF}_{3}+(\mathrm{M}) \longrightarrow 2 \mathrm{CF}_{3}+(\mathrm{M})
$$

wird die Hochdruckgeschwindigkeitskonstante für den $\mathrm{C}_{2} \mathrm{~F}_{6}$-Zerfall erhalten. Da die Bindungsenergie von $\mathrm{CF}_{3}-\mathrm{CF}_{3}$ mit $410 \mathrm{~kJ} \mathrm{~mol}^{-1}$ (bei $0 \mathrm{~K}$ ) praktisch identisch mit dem Wert von $\mathrm{C}_{2} \mathrm{HF}_{5}$ ( [36] und Tabelle 4.1] ist, kann angenommen werden, dass die limitierenden Hochdruck-Dissoziationsgeschwindigkeitskonstanten ebenfalls beinahe gleich sind. Das heißt, es wird angenommen, dass $k_{4.8, \infty}$ durch den Wert

$$
k_{4.9, \infty}=2.2 \cdot 10^{18} \cdot \exp \left(-410 \mathrm{~kJ} \mathrm{~mol}^{-1} / R T\right) \mathrm{s}^{-1}
$$

aus [20] für $\mathrm{C}_{2} \mathrm{~F}_{6}$ gegeben ist.

Beruhend auf Falloff-Effekten ist dabei zu beachten, dass für Reaktion (4.8), bei den vorliegenden experimentellen Bedingungen, eine Verringerung von $k_{4.8} \mathrm{zu}$ Werten, die niedriger sind als $k_{4.8, \infty}$, zu erwarten ist. Die Modellierung [38] der limitierenden Niederdruckgeschwindigkeitskonstante $k_{4.8,0}$ führt zu beinahe identischen Ergebnissen, wie für die Reaktion (4.13) [28]:

$$
\left.k_{4.8,0}=4.3 \cdot 10^{34}(T / 300 \mathrm{~K})^{-13.8} \cdot \exp -410 \mathrm{~kJ} \mathrm{~mol}^{-1} / R T\right) \mathrm{cm}^{3} \mathrm{~mol}^{-1} \mathrm{~s}^{-1}
$$

Darüber hinaus werden Verbreiterungsfaktoren [38-40] für die Falloff-Kurven bei $1300-2000 \mathrm{~K}$ abgeschätzt mit $F_{c e n t, 4.8} \approx 0.04$. Dies bedeutet (siehe auch Abb. 3 in [28]), dass $k_{4.8}$ um etwa den Faktor 3 niedriger liegt, als $k_{4.8, \infty}$ (bei $[A r]=$ $5 \cdot 10^{-5} \mathrm{~mol} \mathrm{~cm}^{-3}$ und $\mathrm{T}=1300 \mathrm{~K}$ ), während bei Temperaturen unter $\mathrm{T}=1500 \mathrm{~K}$ der Faktor auf 5 steigt und sogar auf einen Faktor von 10 unter $\mathrm{T}=1800 \mathrm{~K}$. Diese 
Ergebnisse werden in Abb. 4.3 zusammengefasst und stimmen gut mit den vorliegen Ergebnissen für niedrige Konzentrationen (und damit $k_{\mathrm{C}_{2} \mathrm{HF}_{5}, l}$ ) überein. Beachtet werden sollte bei der Abbildung, dass die durchgezogene Linie aufgrund von verschiedenen Falloff-Effekten bei unterschiedlichen Temperaturen nicht-linear ist (siehe auch [19]).

Die beschrieben Falloff-Effekte erklären auch gut die gegensätzlichen Schlussfolgerungen über die Dominanz der HF-Eliminiation (4.4) [5] und die des C-C-Bindungsbruchs in dieser Arbeit bzw. [9]. Bei $10^{-4}$ Torr und $1300 \mathrm{~K}$ liegen beide Reaktionen (4.4 und 4.8) nahe ihren Niederdruck-Grenzen. Dort wird $k_{4.4,0}$ aufgrund der kleineren Energiebarriere und der Verarmung der höheren Reaktionskanäle bei Zwei-Kanalunimolekularen Reaktionen bei niedrigen Drücken [19] signifikant größer als $k_{4.8,0}$. Eine Möglichkeit wäre es, diesen Verarmungseffekt durch Kombination von [29] und [43] zu modellieren, um abschätzen zu können, bei welcher Temperatur im Niederdruckbereich die primären Zerfallsprozesse des Radikalmechanismus über die HF-Elimination dominieren.

Dies wird im Rahmen dieser Arbeit jedoch nicht durchgeführt, da unter praktischen Gesichtspunkten wie z.B. der Brandbekämpfung nur Hochdruckgeschwindigkeitskonstanten von Interesse sind. Dort ist $k_{4.4, \infty}$ jedoch wesentlich kleiner als $k_{4.8, \infty}$, zumindest für Temperaturen von $\mathrm{T}>850 \mathrm{~K}$ (siehe Gleichung 4.10 und 4.14). Dies führt dazu, dass auch die Produktausbeuten von $\mathrm{C}_{2} \mathrm{HF}_{5}$ aus [5] nicht für praktische Anwendungen berücksichtigt werden sollten.

\subsubsection{Modellierung des Sekundärmechanismus}

Im vorherigen Kapitel konnte gezeigt werden, dass bei den vorliegenden experimentellen Bedingungen bei niedrigen Reaktandkonzentrationen die Dissoziation durch den primären Schritt 4.8, d.h. den C-C-Bindungsbruch, eingeleitet wird. Die daraus abgeleitete Geschwindigkeitskonstante stimmt mit der von Reaktion $\mathrm{C}_{2} \mathrm{~F}_{6} \longrightarrow 2 \mathrm{CF}_{3}$ überein. Beide Reaktionen liegen energetisch nahe beieinander. Es ist daher anzunehmen, dass $k_{4.8}$ mit der Geschwindigkeitskonstante des $\mathrm{C}_{2} \mathrm{~F}_{6}$ Zerfalls übereinstimmt. Dieser wurde druck- und temperaturabhängig bereits in [20] untersucht und druckabhängige Arrhenius-Ausdrücke formuliert (siehe Abb. (4.2), bzw. [20]). Die $\mathrm{CF}_{2}$-Bildung erfolgt dann im nächsten Schritt über die Reaktion $\mathrm{CF}_{2} \mathrm{H}+(\mathrm{M}) \longrightarrow \mathrm{CF}_{2}+\mathrm{H}+(\mathrm{M})$ und den langsameren Zerfall von $\mathrm{CF}_{3}$ :

$$
\mathrm{CF}_{3}+(\mathrm{M}) \longrightarrow \mathrm{CF}_{2}+\mathrm{F}+(\mathrm{M})
$$

Daraus ergibt sich folgende Gesamtreaktion für den $\mathrm{C}_{2} \mathrm{HF}_{5}$-Zerfall: 


$$
\mathrm{C}_{2} \mathrm{~F}_{5} \mathrm{H} \longrightarrow 2 \mathrm{CF}_{2}+\mathrm{H}+\mathrm{F}
$$

Reaktion (4.16) wurde in [28] untersucht. Die Reaktion liegt nahe dem Niedrigdruckgrenzbereich für die unimolekulare Dissoziation mit einer Geschwindigkeitskonstante von:

$$
k_{4.16}=[A r] 3.5 \cdot 10^{15} \cdot \exp \left(-249.6 \mathrm{~kJ} \mathrm{~mol}{ }^{-1} / R T\right) \mathrm{cm}^{3} \mathrm{~mol}^{-1} \mathrm{~s}^{-1}
$$

Bei einer Temperatur von $1500 \mathrm{~K}$ und Drücken von $[\mathrm{Ar}]=10^{-4} \mathrm{~mol} \mathrm{~cm} \mathrm{c}^{-3}$ ergibt sich $k_{4.16}=7 \cdot 10^{2} \mathrm{~s}^{-1}$, während $k_{4.8}=3 \cdot 10^{3} \mathrm{~s}^{-1}$ ist. Andererseits beträgt die $\mathrm{F}_{2} \mathrm{C}-\mathrm{H}$ Bindungsenergie bei $0 \mathrm{~K}_{2} 260 \mathrm{~kJ} \mathrm{~mol}^{-1}$ (aus [36], bzw. $262.1 \mathrm{~kJ} \mathrm{~mol}^{-1}$ auf Basis des G4MP2 Niveaus) und ist damit beträchtlich kleiner als die von $\mathrm{F}_{2} \mathrm{C}-\mathrm{F}$, welche $350 \mathrm{~kJ} \mathrm{~mol}^{-1}$ beträgt ( [36 bzw. $350.7 \mathrm{~kJ} \mathrm{~mol}^{-1}$ auf Basis des G4MP2 Niveaus). Daher sollte die Reaktion $\mathrm{CF}_{2} \mathrm{H}+(\mathrm{M}) \longrightarrow \mathrm{CF}_{2}+\mathrm{H}+(\mathrm{M})$ viel schneller sein, als Reaktion (4.8) und sofort zu einem $\mathrm{CF}_{2}$ führen, während das zweite $\mathrm{CF}_{2}$ auf einer langsameren Zeitskala gebildet wird. Einige Hinweise für die verspätete Bildung des zweiten $\mathrm{CF}_{2}$ sind wahrscheinlich am unteren Ende des in dieser Arbeit betrachteten Temperaturbereichs sichtbar.

Der beschriebene Radikalmechanismus für $\mathrm{C}_{2} \mathrm{HF}_{5}$ bei höheren Reaktandenkonzentrationen $(1000 \mathrm{ppm})$ zeigt eine unerwartete langsamere Bildung von $\mathrm{CF}_{2}$, während die Gesamtausbeute gleich bleibt, bei zwei $\mathrm{CF}_{2}$ pro $\mathrm{C}_{2} \mathrm{HF}_{5}-$ Molekül. Quantenchemische Rechnungen können helfen, den Ursprung dieses Konzentrationseffektes zu identifizieren. Endotherme Reaktionen wie, z.B.

$$
\mathrm{CF}_{2}+\mathrm{CF}_{3} \mathrm{CF}_{2} \mathrm{H} \longrightarrow \mathrm{CF}_{2} \mathrm{H}+\mathrm{C}_{2} \mathrm{~F}_{5}
$$

mit $\Delta H_{0}^{0} \approx 167.3 \mathrm{~kJ} \mathrm{~mol}^{-1}$ (auf dem G3MP2B3 Niveau) und

$$
\mathrm{CF}_{2}+\mathrm{CF}_{3} \mathrm{CF}_{2} \mathrm{H} \longrightarrow \mathrm{CF}_{3}+\mathrm{CF}_{3} \mathrm{CFH}
$$

mit $\Delta H_{0}^{0}=109.7 \mathrm{~kJ} \mathrm{~mol}^{-1}$ (auf dem G4MP2 Level) können bei den vorliegenden niedrigen Reaktandenkonzentrationen wahrscheinlich ausgeschlossen werden. Andererseits könnte eine kurze Kettenreaktion in Betracht gezogen werden, welche mit der folgenden Reaktion beginnt: 


$$
\mathrm{H}+\mathrm{CF}_{3} \mathrm{CF}_{2} \mathrm{H} \longrightarrow \mathrm{CF}_{3} \mathrm{H}+\mathrm{CF}_{2} \mathrm{H}
$$

mit $\Delta H_{0}^{0}=-48.0 \mathrm{~kJ} \mathrm{~mol}^{-1}$ (auf dem G3MP2B3 Niveau). Darauf folgend schließt sich die Reaktion $\mathrm{CF}_{2} \mathrm{H}+(\mathrm{M}) \longrightarrow \mathrm{CF}_{2}+\mathrm{H}+(\mathrm{M})$ an, welche erneut $\mathrm{H}$-Atome bildet und $\mathrm{C}_{2} \mathrm{HF}_{5}$ in $\mathrm{CF}_{2}$ und $\mathrm{CF}_{3} \mathrm{H}$ überführt. Darüber hinaus setzt diese Reaktion das zweite $\mathrm{CF}_{2}$ mittels $\mathrm{CF}_{3} \mathrm{H}$-Zerfalls (Reaktion (4.3)) frei.

Die vorliegenden Rechnungen zeigen jedoch, dass Reaktion (4.21) eine große Barriere besitzt $\left(\Delta H_{0}^{0}=224.9 \mathrm{~kJ} \mathrm{~mol}^{-1}\right.$ auf dem G3MP2B3 Niveau, bzw. $\Delta H_{0}^{0}=252.0 \mathrm{~kJ} \mathrm{~mol}^{-1}$ auf dem CBS-QB3 Niveau) und damit ausgeschlossen werden kann.

Als Alternative bietet sich die folgende nahezu thermoneutrale $\left(\Delta H_{298}^{0}=-2.2 \mathrm{~kJ} \mathrm{~mol}^{-1}\right)$ Reaktion an:

$$
\mathrm{H}+\mathrm{CF}_{3} \mathrm{CF}_{2} \mathrm{H} \longrightarrow \mathrm{H}_{2}+\mathrm{C}_{2} \mathrm{~F}_{5}
$$

welche mit den Reaktionen:

$$
\mathrm{C}_{2} \mathrm{~F}_{5}+(\mathrm{M}) \longrightarrow \mathrm{CF}_{2}+\mathrm{CF}_{3}+(\mathrm{M})
$$

und

$$
\mathrm{CF}_{3}+\mathrm{CF}_{3} \mathrm{CF}_{2} \mathrm{H} \longrightarrow \mathrm{CF}_{3} \mathrm{H}+\mathrm{C}_{2} \mathrm{~F}_{5}
$$

eine $\mathrm{CF}_{3} / \mathrm{C}_{2} \mathrm{~F}_{5}$-Kette bildet, die $\mathrm{zu} \mathrm{CF}_{3} \mathrm{H}$ führt.

Es existieren nur sehr unvollständige Daten für Reaktion (4.24) [44|. Deshalb wird die Geschwindigkeitskonstante von Reaktion (4.24) mittels CBS-QB3-Berechnungen und Transition State Theory zu $k_{4.24} \approx 1.9 \cdot 10^{11} \mathrm{~cm}^{-3} \mathrm{~mol}^{-1} \mathrm{~s}^{-1}(1500 \mathrm{~K})$ und $k_{4.24} \approx$ $1.3 \cdot 10^{12} \mathrm{~cm}^{-3} \mathrm{~mol}^{-1} \mathrm{~s}^{-1}(2000 \mathrm{~K})$ ermittelt. Desgleichen wird $k_{4.22} \approx 1 \cdot 10^{13} \mathrm{~cm}^{-3} \mathrm{~mol}^{-1} \mathrm{~s}^{-1}$ $(1500 \mathrm{~K})$ und $k_{4.22} \approx 2.4 \cdot 10^{13} \mathrm{~cm}^{-3} \mathrm{~mol}^{-1} \mathrm{~s}^{-1}(2000 \mathrm{~K})$ bestimmt. Unabhängig davon, ob $\mathrm{CF}_{3}$ durch die Primärreaktion (4.8) oder die Folge von (4.22) und (4.23) gebildet wird, würde der vorgeschlagene Mechanismus bei hohen Konzentrationen die $\mathrm{CF}_{3} \mathrm{H}$-Bildung plausibel erklären. Beispielsweise würde sich bei $1500 \mathrm{~K}$ und $1000 \mathrm{ppm}$ Reaktandenkonzentration eine Geschwindigkeitskonstante pseudo-erster-Ordnung von $1.7 \cdot 10^{4} \mathrm{~s}^{-1}$ ergeben. 
Eine weitere Möglichkeit wäre es, einen Kettenmechanismus zu formulieren, der über F-Atome verläuft. Fluoratome könnten durch Reaktion (4.16) gebildet werden und über

$$
\mathrm{F}+\mathrm{C}_{2} \mathrm{~F}_{5} \mathrm{H} \longrightarrow \mathrm{HF}+\mathrm{C}_{2} \mathrm{~F}_{5}
$$

ein $\mathrm{H}$-Atom vom $\mathrm{C}_{2} \mathrm{HF}_{5}$ abstrahieren. Als Folge könnten die Reaktionen (4.23) und (4.24) auftreten und wiederum eine $\mathrm{CF}_{3} \mathrm{H}$-bildende Kette beginnen. Geschwindigkeitskonstanten für Reaktion (4.25) können aus [45] und [46] abgeleitet werden. Dieser Reaktionsweg könnte für hohe Konzentrationen (1000 ppm) möglich sein.

Es muss an dieser Stelle erwähnt werden, dass der vorgeschlagene Mechanismus vorläufig ist, auch wenn dieser alle Aspekte der beobachteten $\mathrm{CF}_{2}$-Experimente beschreibt. Sowohl die Produktausbeuten von zwei $\mathrm{CF}_{2}$ pro $\mathrm{C}_{2} \mathrm{HF}_{5}-$ Molekül, als auch die herabgesetzte Geschwindigkeit der $\mathrm{CF}_{2}$-Bildung bei höheren Konzentrationen von $\mathrm{C}_{2} \mathrm{HF}_{5}$ durch bimolekulare Reaktionen von Intermediaten (wahrscheinlich $\mathrm{H}$-Atome) lassen sich so verstehen. Auch der Übergang von einer höheren Geschwindigkeitskonstanten für den $\mathrm{C}_{2} \mathrm{HF}_{5}$-Zerfall bei niedrigen Konzentrationen, welcher nahe an der $\mathrm{C}_{2} \mathrm{~F}_{6}$-Zerfallsrate liegt, zu einer Geschwindigkeitskonstanten bei höheren Konzentrationen in der Größe der $\mathrm{CF}_{3} \mathrm{H}$-Zerfallsrate, wird damit erklärt. Die Endprodukte sind unter den vorliegenden Bedingungen dann $\mathrm{CF}_{2}, \mathrm{HF}$ und $\mathrm{H}_{2}$, sowie $\mathrm{H}$ - und F-Atome, welche auf längeren Zeitskalen rekombinieren.

\subsection{Resultate zur Pyrolyse des Heptafluorethans}

Die Experimente mit $\mathrm{C}_{3} \mathrm{HF}_{7}$ sehen sowohl in ihrer Arrhenius-Auftragung, als auch bei den Einzelschussmessungen denen mit $\mathrm{C}_{2} \mathrm{HF}_{5}$ ähnlich. Auch in diesem Fall zeigt sich eine Konzentrationsabhängigkeit der Geschwindigkeitskonstanten, was einen Mechanismus analog zum $\mathrm{C}_{2} \mathrm{HF}_{5}$-System nahelegt.

Die Stoßwellenuntersuchungen aus [3] nehmen den schon erwähnten 68-Schritt Reaktionsmechanismus an, deren initiale Schritte wie folgt aussehen:

$$
\begin{aligned}
& \mathrm{CF}_{3} \mathrm{CHFCF}_{3}+(\mathrm{M}) \longrightarrow \mathrm{C}_{3} \mathrm{~F}_{6}+\mathrm{HF}+(\mathrm{M}) \\
& \mathrm{CF}_{3} \mathrm{CHFCF}_{3}+(\mathrm{M}) \longrightarrow \mathrm{CF}_{3} \mathrm{CHF}+\mathrm{CF}_{3}+(\mathrm{M})
\end{aligned}
$$




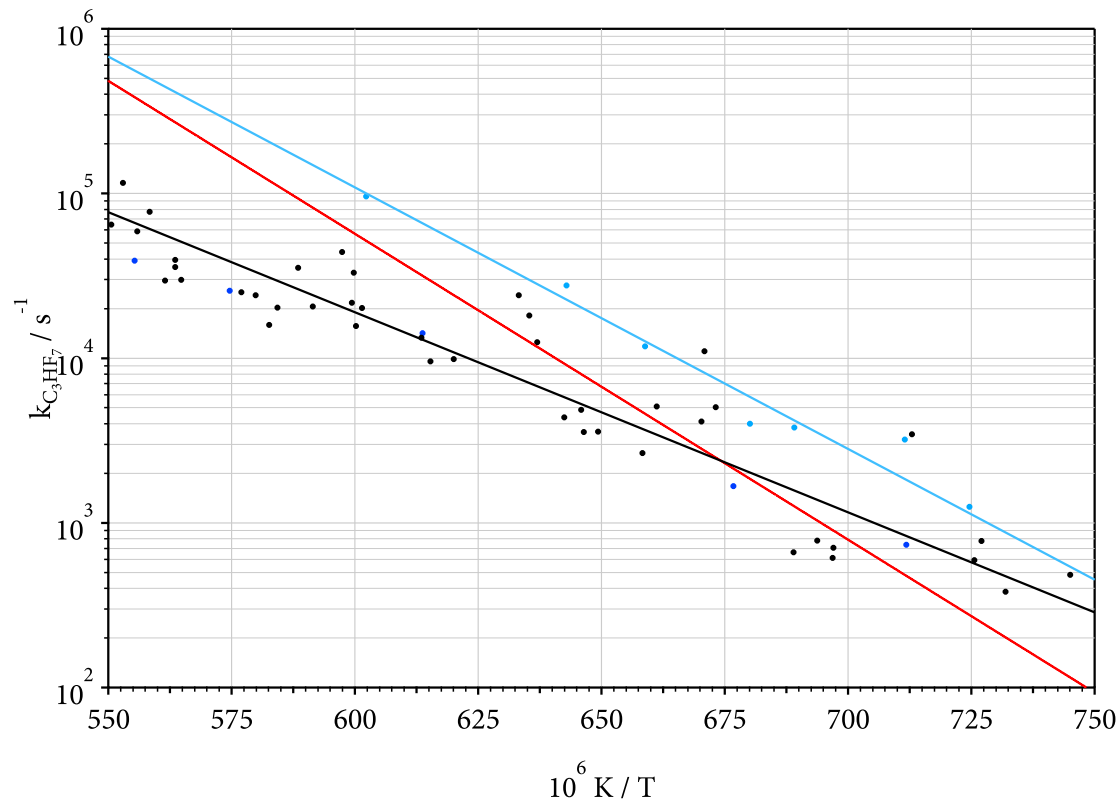

Abb. 4.5: Arrheniusauftragung der effektiven Geschwindigkeitskonstante der Pyrolyse von $\mathrm{C}_{3} \mathrm{HF}_{7}: \bullet 70 \mathrm{ppm} ; \bullet 1000 \mathrm{ppm} ; \cdot 500 \mathrm{ppm}$; blaue Linie: $k_{\mathrm{C}_{3} \mathrm{HF}_{7}, l}$ (Gl. 3.7), schwarze Linie: $k_{\mathrm{C}_{3} \mathrm{HF}_{7}, h}(\mathrm{Gl} .3 .9)$, rote Linie: $k\left(\mathrm{C}_{3} \mathrm{HF}_{7} \longrightarrow \mathrm{CF}_{3} \mathrm{CHF}+\mathrm{CF}_{3}\right)[3]$

sowie

$$
\mathrm{CF}_{3} \mathrm{CHFCF}_{3}+(\mathrm{M}) \longrightarrow \mathrm{CF}_{3} \mathrm{CFCF}_{3}+\mathrm{H}+(\mathrm{M})
$$

Mittels der in dieser Arbeit genutzten UV-Absorptionsspektroskopie von $\mathrm{CF}_{2}$ könnten die Reaktionen (4.26) und (4.27) gut nachweisbar sein, da $\mathrm{CF}_{2}$ direkt aus $\mathrm{C}_{3} \mathrm{~F}_{6}$ oder $\mathrm{CF}_{3} \mathrm{CHF}$ gebildet werden kann. Ähnlich dem $\mathrm{C}_{2} \mathrm{HF}_{5}-$ Zerfall liegt es nahe, einen Mechanismus anzunehmen, bei dem aus $\mathrm{C}_{3} \mathrm{HF}_{7}$ in einem ersten Reaktionsschritt $\mathrm{C}_{3} \mathrm{~F}_{6}$ gebildet wird, welches schnell zu $\mathrm{CF}_{2}$ zerfällt. Die vorliegenden Untersuchungen zeigen jedoch, dass auch der Pyrolyse von Heptafluorethan ein komplexerer Radikalmechanismus zu Grunde liegen muss.

Der direkte Vergleich (Abb. 4.5) der Geschwindigkeitskonstanten von niedriger und hoher Konzentration von $\mathrm{C}_{3} \mathrm{HF}_{7}$ zeigt ein ähnliches Verhalten wie beim $\mathrm{C}_{2} \mathrm{HF}_{5}$. Es scheint ebenfalls ein Übergang zwischen den Geschwindigkeitskonstanten hoher und 


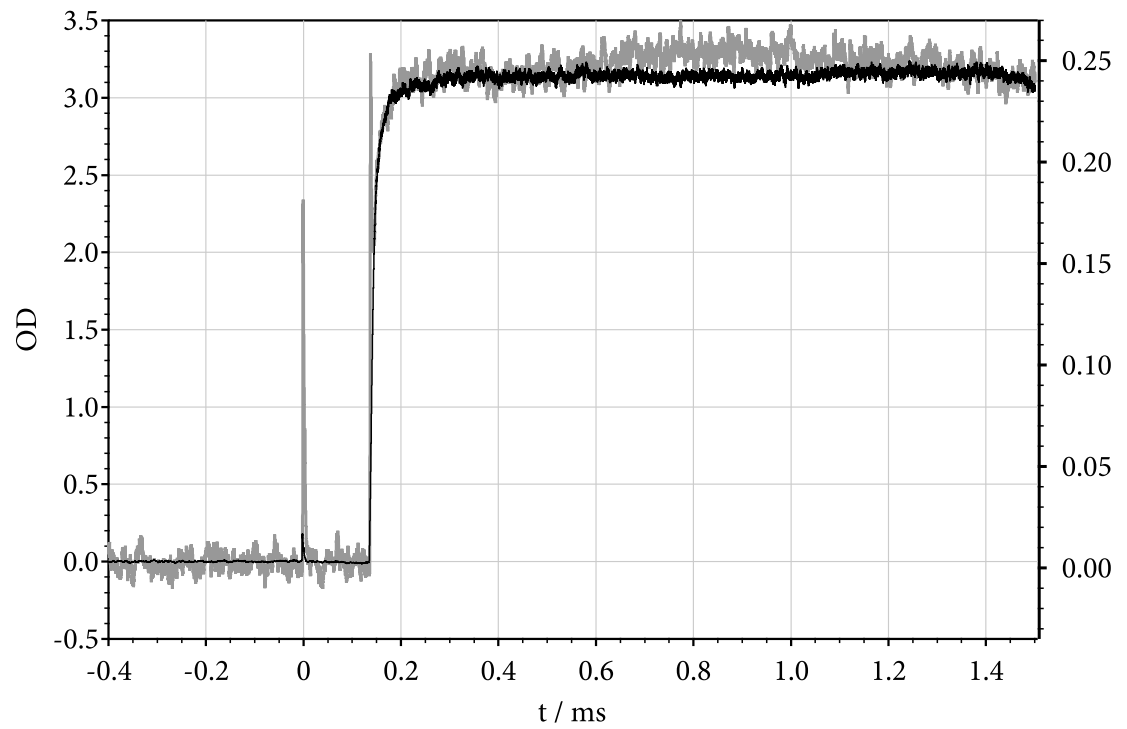

Abb. 4.6: Vergleich zweier Einzelschussmessungen von $\mathrm{C}_{3} \mathrm{HF}_{7}$. Graue Linie: $70 \mathrm{ppm}$, schwarze Linie: 900 ppm bei etwa $2000 \mathrm{~K}$

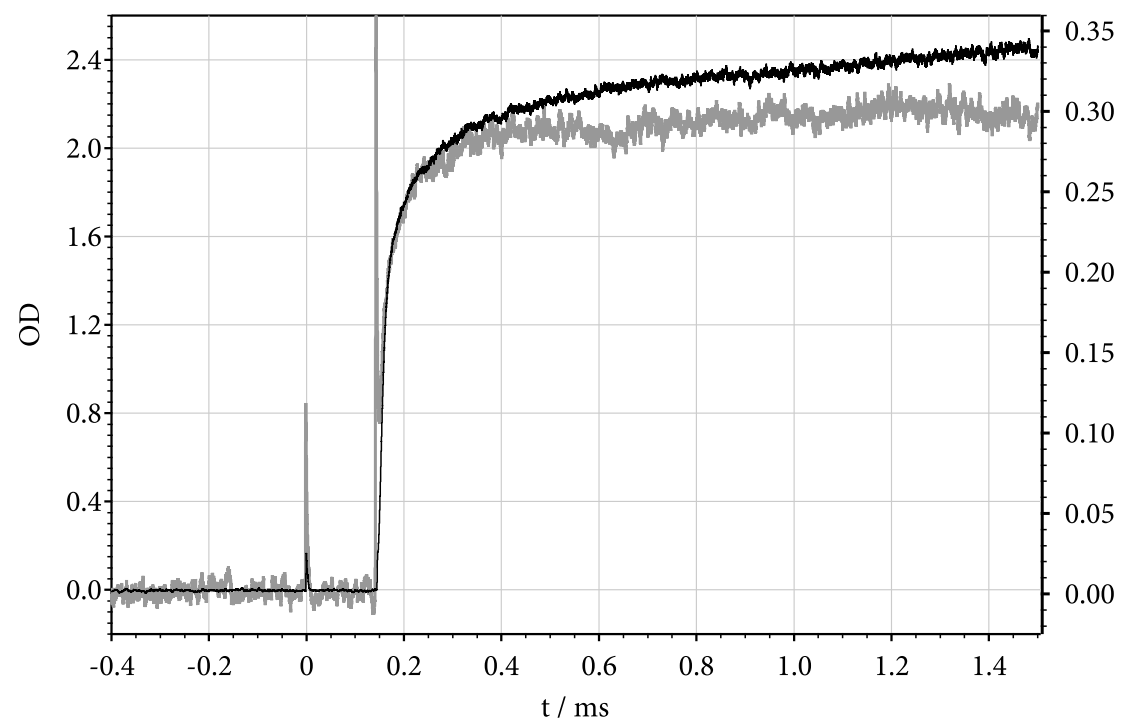

Abb. 4.7: Vergleich zweier Einzelschussmessungen von $\mathrm{C}_{3} \mathrm{HF}_{7}$. Graue Linie: $70 \mathrm{ppm}$, schwarze Linie: 500 ppm bei etwa $1750 \mathrm{~K}$ 
niedriger Reaktandenkonzentrationen zu existieren. Die Geschwindigkeitskonstante für niedrige Konzentrationen liegt näher bei der für Reaktion (4.2) $\left(\mathrm{C}_{2} \mathrm{~F}_{6} \longrightarrow 2 \mathrm{CF}_{3}\right)$, während $k$ für hohe Konzentrationen näher bei der für Reaktion $(4.3)\left(\mathrm{CF}_{3} \mathrm{H} \longrightarrow\right.$ $\mathrm{CF}_{2}+\mathrm{HF}$ ) liegt. Weiterhin fällt auf, dass $k_{\mathrm{C}_{3} \mathrm{HF}_{7}, l}$ um etwa einen Faktor 10 größer ist, als $k_{\mathrm{C}_{2} \mathrm{HF}_{5}, l}$.

Dabei muss noch einmal darauf hingewiesen werden, dass $k_{\mathrm{C}_{3} \mathrm{HF}_{7}, l}$ und $k_{\mathrm{C}_{3} \mathrm{HF}_{7}, h}$ ebenso wie $k_{\mathrm{C}_{2} \mathrm{HF}_{5}, l}$ und $k_{\mathrm{C}_{2} \mathrm{HF}_{5}, h}$ lediglich zusammengesetzte Geschwindigkeitskonstanten sind, in denen auch Effekte von sekundären Reaktionen eine Rolle spielen.

Quantenchemische Rechnungen der primären Reaktionsschritte von $\mathrm{C}_{3} \mathrm{HF}_{7}$ sind bereits in [47] durchgeführt worden. Von Carlos Cobos werden diese Rechnungen auf weitere mögliche primäre, wie auch sekundäre, Reaktionsschritte ausgedehnt.

\subsubsection{Modellierung der primären Reaktionsschritte}

Für die verschiedenen möglichen Zerfallsschritte des $\mathrm{C}_{3} \mathrm{HF}_{7}$ werden die schon in Abschnitt 4.1.1 genannten quantenchemischen Rechnungen durchgeführt, um die Thermochemie und die Energiebarrieren zu bestimmen. Die Tabellen 4.3 und 4.4 fassen die Resultate zusammen. Folgende Reaktionswege werden berücksichtigt:

$$
\begin{aligned}
& \mathrm{CF}_{3} \mathrm{CHFCF}_{3} \longrightarrow \mathrm{CF}_{3} \mathrm{CFCF}_{2}+\mathrm{HF} \\
& \mathrm{CF}_{3} \mathrm{CHFCF}_{3} \longrightarrow \mathrm{CF}_{3} \mathrm{CCF}_{3}+\mathrm{HF} \\
& \mathrm{CF}_{3} \mathrm{CHFCF}_{3} \longrightarrow \mathrm{CF}_{3} \mathrm{H}+\mathrm{CF}_{3} \mathrm{CF} \\
& \mathrm{CF}_{3} \mathrm{CHFCF}_{3} \longrightarrow \mathrm{CF}_{3} \mathrm{CFH}+\mathrm{CF}_{3}
\end{aligned}
$$

Die Reaktionen (4.29), (4.30) und (4.31) sind Eliminationsreaktionen mit starren aktivierten Komplexen, während der zu bevorzugende, einfache Bindungsbruch durch Reaktion (4.32) dargestellt wird. Ebenso wie für das $\mathrm{C}_{2} \mathrm{HF}_{5}$ muss die temperaturund druckabhängige Konkurrenz zwischen Reaktionen mit starren und lockeren 
Tab. 4.3: Reakionsenthalpien für die Zerfallskanäle von $\mathrm{C}_{3} \mathrm{HF}_{7}$ bei $0 \mathrm{~K}$ in kJ mol${ }^{-1}$

\begin{tabular}{l|cccccc}
\hline Reaktionsprodukt & BMK & MO6-2X & CBS-QB3 & G3MP2B3 & G4 & Table \\
& 30 & {$[31$} & $\mid 32$ & 33 & 34 & 47 \\
\hline $\mathrm{CF}_{3} \mathrm{CFCF}_{2}+\mathrm{HF}\left({ }_{4} \cdot 29\right)$ & 138 & 141 & 134 & 130 & 128 & 146 \\
$\mathrm{CF}_{3} \mathrm{CCF}_{3}+\mathrm{HF}\left({ }_{4} \cdot 30\right)$ & 409 & 410 & 407 & 393 & 387 & 401 \\
$\left.\mathrm{CHF}_{3}+\mathrm{CF}_{3} \mathrm{CF}_{4} \cdot 31\right)$ & 341 & 355 & 353 & 348 & 339 & 343 \\
$\mathrm{CF}_{3} \mathrm{CFH}+\mathrm{CF}_{3}\left({ }_{4} \cdot 32\right)$ & 377 & 388 & 399 & 393 & 370 & 386 \\
\hline
\end{tabular}

Tab. 4.4: Barrierenhöhen für die Zerfallskanäle von $\mathrm{C}_{3} \mathrm{HF}_{7}$ bei $0 \mathrm{~K}$ (bzw. bei $298 \mathrm{~K}$ in $\mathrm{kJ}$ $\mathrm{mol}^{-1} \mid 47$

\begin{tabular}{|c|c|c|c|c|c|c|}
\hline Reaktionsprodukt & $\begin{array}{c}\text { BMK } \\
30\end{array}$ & $\begin{array}{c}\text { MO6-2X } \\
31\end{array}$ & $\begin{array}{c}\text { CBS-QB3 } \\
\mid 32\end{array}$ & $\begin{array}{c}\text { G3MP2B3 } \\
33\end{array}$ & $\begin{array}{l}\text { G4 } \\
34\end{array}$ & $\begin{array}{c}\text { Table } \\
47\end{array}$ \\
\hline $\left.\mathrm{CF}_{3} \mathrm{CFCF}_{2}+\mathrm{HF}_{4} \cdot 29\right)$ & 322 & 325 & 329 & 326 & 326 & 333 \\
\hline $\left.\mathrm{CF}_{3} \mathrm{CCF}_{3}+\mathrm{HF}_{4} \cdot 30\right)$ & - & - & - & - & - & 371 \\
\hline $\mathrm{CHF}_{3}+\mathrm{CF}_{3} \mathrm{CF}\left({ }_{4} \cdot 31\right)$ & - & - & - & - & - & 408 \\
\hline $\mathrm{CF}_{3} \mathrm{CFH}+\mathrm{CF}_{3}\left({ }_{4} \cdot 32\right)$ & 377 & 388 & 399 & 393 & 370 & 386 \\
\hline
\end{tabular}

aktivierten Komplexen diskutiert werden. Die Ähnlichkeit der Konzentrationsabhängigkeit der Geschwindigkeitskonstanten $k_{\mathrm{C}_{2} \mathrm{HF}_{5}}$ und $k_{\mathrm{C}_{3} \mathrm{HF}_{7}}$ lässt darauf schließen, dass eine Dominanz des radikal-bildenden C-C-Bindungsbruchs vorliegt (4.32). Dies gilt zumindest für den beobachteten Temperatur- $(1300$ - $1900 \mathrm{~K})$ und Druckbereich. Im Vergleich zu Reaktion (4.32) ist die Bindungsenergie von Reaktion (4.8) um $18 \mathrm{~kJ} \mathrm{~mol}^{-1}$ (bei $0 \mathrm{~K}$ ) höher, was den Faktor 10 erklärt, der zwischen $k_{\mathrm{C}_{3} \mathrm{HF}_{7}, l}$ und $k_{\mathrm{C}_{2} \mathrm{HF}_{5},}$ liegt. Reaktion (4.32) liegt daher auch näher an der Hochdruckgrenze der unimolekularen Bindungsbruchreaktion, als (4.8). Es liegt also, wie auch beim $\mathrm{C}_{2} \mathrm{HF}_{5}$, eine Dominanz von Reaktion (4.32) über die Reaktionen (4.29) - (4.31) vor. Dies passt zu den Ergebnissen von [3], bei denen sich die Geschwindigkeitskonstanten wie folgt ergaben:

$$
\begin{aligned}
& k_{4.29}=10^{12.9} \cdot \exp (-35000 \mathrm{~K} / T) \mathrm{s}^{-1} \\
& k_{4.32}=10^{15.7} \cdot \exp (-42800 \mathrm{~K} / T) \mathrm{s}^{-1}
\end{aligned}
$$


Daraus lässt sich schließen, dass Reaktion (4.32) bei Temperaturen unter $1120 \mathrm{~K}$ langsamer als Reaktion (4.29) sein sollte. Allerdings fanden sich in [5] keine Beweise für die Existenz von Reaktion (4.32) bei Temperaturen zwischen 900 und $1500 \mathrm{~K}$. Statt dessen wurde vorgeschlagen, dass Reaktion (4.29) bis etwa $1200 \mathrm{~K}$ dominiert, um dann von Reaktion (4.30) abgelöst zu werden.

Diese unterschiedlichen Schlussfolgerungen werden vereinbar, wenn man die großen Druckdifferenzen zwischen den zwei Arbeiten berücksichtigt. Während bei [3] mit Drücken von 16 - 18 atm gearbeitet wurde, waren es bei [5] $10^{-4}-10^{-5}$ Torr. Wie schon beim $\mathrm{C}_{2} \mathrm{HF}_{5}$ beschrieben, reduzieren Falloff-Effekte bei einer unimolekularen Reaktion mit zwei Kanälen bei fallenden Drücken die Geschwindigkeitskonstante des energetisch höheren Kanals. Die Produktausbeuten aus [5] sind daher nicht für praktische Anwendungen im Hochdruckfall zu verwenden.

In dieser Arbeit werden auch die Hochdruck-Grenzgeschwindigkeitskonstanten der Reaktionen (4.29) - (4.31) mit Hilfe der Charakteristika der Übergangszustände und 47] modelliert. Für einen Temperaturbereich von 1500 - $2500 \mathrm{~K}$ erhält man aus G4MP2 Berechnungen:

$$
k_{4.30}=3.6 \cdot 10^{15} \cdot \exp (-44300 \mathrm{~K} / T) \mathrm{s}^{-1}
$$

während man aus den ab initio Ergebnissen von [4]

$$
k_{4.31}=5.8 \cdot 10^{14} \cdot \exp (-48600 \mathrm{~K} / T) \mathrm{s}^{-1}
$$

und

$$
k_{4.32}=3.5 \cdot 10^{14} \cdot \exp (-53000 \mathrm{~K} / T) \mathrm{s}^{-1}
$$

bestimmen kann. Alle Geschwindigkeitskonstanten liegen damit weit unterhalb des berechneten Werte für $k_{\mathrm{C}_{3} \mathrm{HF}_{7}}$ und bestätigen so die Annahme, dass die Bindungsbruchreaktion (4.32) bei den vorliegenden Bedingungen über die Reaktionen (4.29) (4.31) dominiert. 


\subsubsection{Modellierung des Sekundärmechanismus}

Das vorhergehende Kapitel beschreibt den primären Zerfall von $\mathrm{C}_{3} \mathrm{HF}_{7}$, unter den in dieser Arbeit verwendetenden experimentellen Bedingungen, beginnend mit:

$$
\mathrm{CF}_{3} \mathrm{CHFCF}_{3}+(\mathrm{M}) \longrightarrow \mathrm{CF}_{3}+\mathrm{CF}_{3} \mathrm{CFH}+(\mathrm{M})
$$

Analog zum $\mathrm{C}_{2} \mathrm{HF}_{5}$ könnte $\mathrm{CF}_{3} \mathrm{CFH}$ wie folgt weiterreagieren:

$$
\mathrm{CF}_{3} \mathrm{CFH}+(\mathrm{M}) \longrightarrow \mathrm{CF}_{3} \mathrm{CF}+\mathrm{H}+(\mathrm{M})
$$

$\left(\Delta H_{0}^{0}=403 \mathrm{~kJ} \mathrm{~mol}^{-1}\right)$ und über eine schnelle Sequenz der Reaktionen (4.29) und (1.3) zwei $\mathrm{CF}_{2}$ bilden. Es sind jedoch weitere Zerfallsreaktionen des $\mathrm{CF}_{3} \mathrm{CFH}$ denkbar, z.B. die Bildung von $\mathrm{CF}_{3} \mathrm{H}+\mathrm{CF}\left(\Delta H_{0}^{0}=248 \mathrm{~kJ} \mathrm{~mol}^{-1}\right.$, Energie des starren aktivierten Komplexes: $\left.394 \mathrm{~kJ} \mathrm{~mol}^{-1}\right), \mathrm{CF}_{3}+\mathrm{CFH}\left(\Delta H_{0}^{0}=372 \mathrm{~kJ} \mathrm{~mol}^{-1}\right)$ oder auch $\mathrm{CF}_{2} \mathrm{CHF}+\mathrm{F}$ $\left(\Delta H_{0}^{0}=285 \mathrm{~kJ} \mathrm{~mol}^{-1}\right.$, alle Werte in Klammern auf G4MP2 Niveau). Der energetisch günstigste Zerfall von $\mathrm{CF}_{3} \mathrm{CFH}$, welcher keinen starren aktivierten Komplex beinhaltet, führt $\mathrm{zu} \mathrm{CF} 2 \mathrm{CHF}+\mathrm{F}$. Dabei würde $\mathrm{CF}_{2}$ sehr schnell gebildet werden und weiterhin sollten Reaktionen von F und $\mathrm{CFH}$ in Betracht gezogen werden. Ähnlich wie für den sekundären $\mathrm{C}_{2} \mathrm{HF}_{5}$-Zerfall müssen Reaktionen, welche $\mathrm{H}$-Atome und $\mathrm{CF}_{3}$ involvieren, betrachtet werden.

Bisher ist es nicht möglich, auf Basis der gemessenen $\mathrm{CF}_{2}$-Profile den Mechanismus für den $\mathrm{C}_{3} \mathrm{HF}_{7}$-Zerfall bei niedrigen Konzentrationen eindeutig zu entschlüsseln. Ebenfalls kann nicht zweifelsfrei erklärt werden, wie eine Erhöhung der Reaktandenkonzentration zu einer effektiven Absenkung der Geschwindigkeit der $\mathrm{CF}_{2}$-Bildung führt. Diese ist beim $\mathrm{C}_{3} \mathrm{HF}_{7}$ zwar weniger signifikant als beim $\mathrm{C}_{2} \mathrm{HF}_{5}$, aber trotzdem deutlich sichtbar vorhanden, wobei die Gesamtausbeute bei drei $\mathrm{CF}_{2}$ pro eingesetzem $\mathrm{C}_{3} \mathrm{HF}_{7}-$ Molekül bleibt.

Ein Vergleich mit den Single-Puls-Stoßwellen Experimenten von [2] könnte hilfreich sein. Diese Experimente wurden mit sehr viel höheren Reaktandenkonzentrationen (5000 - 30000 ppm) durchgeführt und eine große Anzahl verschiedener Reaktionsprodukte konnte identifiziert werden. Ausführliche kinetische Modellierungen ergeben eine Geschwindigkeitskonstante

$$
k_{4.32}=10^{15.9} \cdot \exp \left(-355.6 \mathrm{~kJ} \mathrm{~mol}^{-1} / T\right) \mathrm{s}^{-1}
$$


im Gegensatz zu

$$
k_{4.29}=10^{12.9} \cdot \exp \left(-291.2 \mathrm{~kJ} \mathrm{~mol}^{-1} / T\right) \mathrm{s}^{-1}
$$

Die vorliegenden Hinweise darauf, dass ein Radikalmechanismus, anstelle eines Mechanismus mit Reaktion (4.29), abläuft, werden durch die Resultate aus [2] unterstützt. $k_{4.32}$ dominiert in einem Temperaturbereich zwischen $1500 \mathrm{~K}$ und $1800 \mathrm{~K}$ über $k_{4.29}$. Es stimmt außerdem mit den Schlussfolgerungen von [37] überein. Die Arbeit von [5], in welcher kein Radikalmechanismus beobachtet werden konnte, ist durch die in diesem Experiment angewandten sehr niedrigen Drücke $\left(<10^{-3}\right.$ Torr $)$ zu erklären.

Damit bleibt die Frage offen, welchen Reaktionen den experimentellen Geschwindigkeitskonstanten $k_{\mathrm{C}_{3} \mathrm{HF}_{7}}$ für hohe und niedrige Konzentrationen aus Gleichungen (3.7) und (3.9) entsprechen. Obwohl die Werte für $k_{\mathrm{C}_{3} \mathrm{HF}_{7}, l}$ (niedrige Konzentration) nahe an der Geschwindigkeitskonstante für $k_{4.29}$ aus [2] liegen, ist es nicht sicher, dass $k_{\mathrm{C}_{3} \mathrm{HF}_{7}, l} \approx k_{4.29}$ entspricht, da die Bildung von $\mathrm{CF}_{2}$ über mehrere mögliche Sekundärreaktionen erfolgen kann, welche wahrscheinlich viel langsamer sind, als Reaktion 4.29. Weiterhin müssen die Werte von $k_{\mathrm{C}_{3} \mathrm{HF}_{7}, h}$ nicht notwendigerweise zu einer verzögerten $\mathrm{CF}_{2}$ Freisetzung aus $\mathrm{CF}_{3} \mathrm{H}$ gehören. Es wären auch andere Zwischenprodukte als „ $\mathrm{CF}_{2}$-Reservoir" möglich, z.B. $\mathrm{C}_{2} \mathrm{HF}_{5}$. Die vorhandenen experimentellen Daten allein sind nicht ausreichend, um sichere Schlussfolgerungen über die Identität der Zwischenprodukte zu liefern. 



\section{Kapitel 5}

\section{Ausblick}

In dieser Arbeit werden die Zerfallsprozesse der fluorierten Kohlenwasserstoffe Pentafluorethan und 2H-Heptafluorethan mittels der bewährten Stoßwellentechnik untersucht.

Die Pyrolyse beider Moleküle wird mit Hilfe von UV-Absorptionsspektroskopie von $\mathrm{CF}_{2}$ bei $248 \mathrm{~nm}$ beobachtet. Dabei wird ein Temperaturbereich von $1200-2000 \mathrm{~K}$ und Drücke zwischen $[\mathrm{Ar}]=2 \cdot 10^{-5}$ und $10 \cdot 10^{-5} \mathrm{~mol} / \mathrm{cm}^{3}$, sowie Reaktandkonzentrationen von etwa $70 \mathrm{ppm}, 500 \mathrm{ppm}$ und $1000 \mathrm{ppm}$ abgedeckt.

Dies ermöglicht es einen detaillierteren Einblick in die primären Reaktionsschritte zu erhalten, als bisherige Arbeiten es vermochten.

Für $\mathrm{C}_{2} \mathrm{HF}_{5}$ kann die Geschwindigkeitskonstante des primären C-C-Bindungsbruchs bestimmt werden, welche nahe an der Geschwindigkeitskonstante für den C-CBindungsbruch des $\mathrm{C}_{2} \mathrm{~F}_{6}$-Zerfalls liegt. Für das Heptafluorpropan kann ein solcher Schluss derzeit nicht eindeutig gezogen werden.

In beiden Fällen war es möglich, den Einfluss der Druckabhängigkeit auf die Geschwindigkeitskonstanten nachzuweisen und auf Basis dessen die unterschiedlichen Ergebnisse vorheriger Arbeiten [3,5, $5,11,48$, zusammenzuführen und zu erklären.

Ein wichtiges Resultat ist, dass die C-C-Bindungsbruchreaktion, welche zu einem Radikalmechanismus führt, bei den vorherrschenden experimentellen Bedingungen immer über die HF-Eliminationsreaktion dominiert. Es kann vermutet werden, dass dies teilweise auch für niedrigere Temperaturen gilt, wie sie in früheren Arbeiten angewandt wurden.

Weiterhin kann die ungewöhnliche Verzögerung der $\mathrm{CF}_{2}$-Bildung bei steigender Reaktandenkonzentration mit Hilfe eines bimolekularen Kettenmechanismus erklärt werden, welcher zu einem intermediärem " $\mathrm{CF}_{2}$-Reservoir" wie $\mathrm{CF}_{3} \mathrm{H}$ führt. Aus diesem kommt es zur langsameren Freisetzung von $\mathrm{CF}_{2}$, als im Reaktionsmechanismus bei kleinen Reaktandenkonzentrationen, wo solche Lagermoleküle nicht gebildet werden. 
Es wäre wünschenwert, wenn die Ergebnisse dieser Arbeit in die Modellierung der Mechanismen Halon-ersetzenden Löschmittel [3] Eingang fänden, da die vorliegenden Messungen bei hohen Drücken ausgeführt wurden, wie sie für praktische Anwendungen von Bedeutung sind.

Obwohl es nicht möglich war, präzise Informationen über die Geschwindigkeitskonstanten einzelner Elementarreaktionen zu erhalten, ergibt sich aus der Kombination der gemessenen und modellierten theoretischen Geschwindigkeitskonstanten ein in sich geschlossenes Bild, welches alle experimentellen Ergebnisse umfasst.

Für die Zukunft ist geplant weitere fluorierte Kohlenwasserstoffe, die einen Einsatz als Kältemittel finden könnten, auf ihren Reaktionsmechanismus hin zu untersuchen. 
Anhang 



\section{Anhang A}

\section{Molekulare Parameter für die Modellierung}

\section{A.1 Harmonische Schwingungsfrequenzen}

$\mathrm{CF}_{3} \mathrm{CF}_{2} \mathrm{H}: v_{i} / \mathrm{cm}^{-1}=3024,1424,1375,1298,1220,1193,1150,1141,861,715,575,570$, $514,407,355,235,201$, und freier Rotor mit $\sigma=3$, und $I_{\text {red }}=36$ amu $\AA^{2}$ aus B3LYP/6$31 \mathrm{G}\left(2 \mathrm{df}, \mathrm{p}\right.$ ) Rechnungen skaliert mit 0.9854 (aus G4 Modell). $v_{i} / \mathrm{cm}^{-1}=3071,1422$, $1369,1290,1191,1173,1122,1121,852,710,570,567,511,407,354,239,206$, und freier Rotor mit $\sigma=3$, und $I_{\text {red }}=36$ amu $\AA^{2}$ aus B3LYP/CBSB7 Rechnungen skaliert mit 0.99 (aus CBS-QB3 Modell).

$\mathrm{CF}_{3} \mathrm{CFHCF}_{3}: v_{i} / \mathrm{cm}^{-1}=3037,1376,1368,1298,1264,1238,1218,1181,1135,1121,901$, $677,599,544,527,509,447,337,318,285,232,220,162$, 91, 22; aus B3LYP/6-31G(2df,p) Rechnungen skaliert mit 0.9854 (aus G4MP2 Modell).

$\mathrm{CF}_{3}: v_{i} / \mathrm{cm}^{-1}=1229$ (2), 1066, 686, 499 (2); aus B3LYP/CBSB7 Rechnungen skaliert mit 0.99 (aus CBS-QB3 Modell).

$\mathrm{CF}_{3} \mathrm{CF}_{2} \mathrm{H} \longrightarrow \mathrm{CF}_{3} \mathrm{CF}+\mathrm{HF}$ : Übergangszustand $v_{i} / \mathrm{cm}^{-1}=2052,1331,1263,1247$, $1158,1039,908,818,673,540,527,415,294,236,219,144$, freier Rotor und 942i; aus B3LYP/6-31G(2df,p) Rechnungen skaliert mit 0.9854 (aus G4 Modell).

$\mathrm{CF}_{3} \mathrm{CF}_{2} \mathrm{H} \longrightarrow \mathrm{CF}_{2} \mathrm{CF}_{2}+\mathrm{HF}$ : Übergangszustand $v_{i} / \mathrm{cm}^{-1}=1725,1536,1436,1263$, $1176,1130,788,725,630,537,498,414,309,256,255,191,103$ und 1785i; aus B3LYP/6$31 \mathrm{G}(2 \mathrm{df}, \mathrm{p})$ Rechnungen skaliert mit 0.9854 (aus G4 Modell).

$\mathrm{CF}_{3} \mathrm{CF}_{2} \mathrm{H} \longrightarrow \mathrm{CF}_{3} \mathrm{H}+\mathrm{CF}_{2}$ : Übergangszustand $v_{i} / \mathrm{cm}^{-1}=2735,1366,1218,1209$, $1196,1172,1039,735,630,581,507,502,263,209,144,142,26$ und 1175i; aus B3LYP/631G(2df,p) Rechnungen skaliert mit 0.9854 (aus G4 Modell).

$\mathrm{CF}_{3} \mathrm{CFHCF}_{3} \longrightarrow \mathrm{CF}_{3} \mathrm{CFCF}_{2}+\mathrm{HF}$ : Übergangszustand $v_{i} / \mathrm{cm}^{-1}=1720,1523,1428$, 1327, 1218, 1200, 1179, 1129, 1013, 787, 763, 700, 608, 589, 536, 495, 412, 367, 340, 277, 261, 236, 197, 161, 76, 51 und 1770i; aus B3LYP/6-31G(2df,p) Rechnungen skaliert mit 0.9854 (aus G4MP2 Modell). 
$\mathrm{CF}_{3} \mathrm{CFHCF}_{3} \longrightarrow \mathrm{CF}_{3} \mathrm{CCF}_{3}+\mathrm{HF}:$ Übergangszustand $v_{i} / \mathrm{cm}^{-1}=3139,1404,1328$, $1295,1262,1223,1212,962,860,736,678,678,596,546,527,518,507,344,319,302$, 286, 205, 171, 155, 95, 29 and 771i; Berechnungen auf dem MP2/6-31G(d) Level aus [47.

$\mathrm{CF}_{3} \mathrm{CFHCF}_{3} \longrightarrow \mathrm{CF}_{3} \mathrm{H}+\mathrm{CF}_{3} \mathrm{CF}$ : Übergangszustand $v_{i} / \mathrm{cm}^{-1}=2121,1446,1349$, $1311,1284,1262,1223,1211,1036,870,807,699,665,564,521,519,515,430,282,254,245$, 195, 185, 128, 81, 52 und 1383i; Berechnungen auf dem MP2/6-31G(d) Level aus [47|.

$\mathrm{H}+\mathrm{CF}_{3} \mathrm{CF}_{2} \mathrm{H} \longrightarrow \mathrm{H}_{2}+\mathrm{C}_{2} \mathrm{~F}_{5}$ : Übergangszustand $v_{i} / \mathrm{cm}^{-1}=1546,1355,1257,1227$, $1203,1168,1158,1095,808,692,591,572,510,413,357,296,294,219,205,58$ und 1359i; aus B3LYP/CBSB7 Rechnungen skaliert mit 0.99 (aus CBS-QB3 Modell).

$\mathrm{CF}_{3}+\mathrm{CF}_{3} \mathrm{CF}_{2} \mathrm{H} \longrightarrow \mathrm{CF}_{3} \mathrm{H}+\mathrm{C}_{2} \mathrm{~F}_{5}$ : Übergangszustand $v_{i} / \mathrm{cm}^{-1}=1458,1449,1309$, $1206,1202,1185,1179,1171,1145,1075,874,740,690,594,573,521,504,503,414,356$, 253, 205, 197, 185, 136, 73, 40, 32, 5 and 1730i; aus B3LYP/CBSB7 Rechnungen skaliert mit 0.99 (aus CBS-QB3 Modell).

\section{A.2 Rotationskonstanten}

$\mathrm{CF}_{3} \mathrm{CF}_{2} \mathrm{H}: \mathrm{A}, \mathrm{B}$ und $\mathrm{C} / \mathrm{cm}^{-1}=0.123,0.081$ und 0.067; aus B3LYP/6-31G(2df,p) Berechnungen (aus G4MP2 Modell). A, B und C/cm-1 = 0.122, 0.080 und 0.066; aus B3LYP/CBSB7 Berechnungen (aus CBS-QB3 Modell).

$\mathrm{CF}_{3} \mathrm{CFHCF}_{3}$ : A, B und $\mathrm{C} / \mathrm{cm}^{-1}=0.070,0.035$ und 0.031; aus B3LYP/6-31G(2df,p) Berechnungen (aus G4MP2 Modell).

$\mathrm{CF}_{3}$ : $\mathrm{A}, \mathrm{B}$ und $\mathrm{C} / \mathrm{cm}^{-1}=0.359,0.359$ und 0.186; aus B3LYP/CBSB7 Berechnungen (aus CBS-QB3 Modell).

$\mathrm{CF}_{3} \mathrm{CF}_{2} \mathrm{H} \longrightarrow \mathrm{CF}_{3} \mathrm{CF}+\mathrm{HF}$ : Übergangszustand $\mathrm{A}, \mathrm{B}$ und $\mathrm{C} / \mathrm{cm}^{-1}=0.109,0.077$ und 0.060; aus B3LYP/6-31G(2df,p) Berechnungen (aus G4 Modell).

$\mathrm{CF}_{3} \mathrm{CF}_{2} \mathrm{H} \longrightarrow \mathrm{CF}_{2} \mathrm{CF}_{2}+\mathrm{HF}$ : Übergangszustand $\mathrm{A}, \mathrm{B}$ und $\mathrm{C} / \mathrm{cm}^{-1}=0.106,0.071$ und 0.065; aus B3LYP/6-31G(2df,p) Berechnungen (aus G4 Modell).

$\mathrm{CF}_{3} \mathrm{CF}_{2} \mathrm{H} \longrightarrow \mathrm{CF}_{3} \mathrm{H}+\mathrm{CF}_{2}$ : Übergangszustand $\mathrm{A}, \mathrm{B}$ und $\mathrm{C} / \mathrm{cm}^{-1}=0.130,0.060$ und 0.051; aus B3LYP/6-31G(2df,p) Berechnungen (aus G4 Modell).

$\mathrm{CF}_{3} \mathrm{CFHCF}_{3} \longrightarrow \mathrm{CF}_{3} \mathrm{CFCF}_{2}+\mathrm{HF}$ : Übergangszustand $\mathrm{A}, \mathrm{B}$ und $\mathrm{C} / \mathrm{cm}^{-1}=0.064$, 0.033 und 0.030; aus B3LYP/6-31G(2df,p) Berechnungen (aus G4MP2 Modell).

$\mathrm{CF}_{3} \mathrm{CFHCF}_{3} \longrightarrow \mathrm{CF}_{3} \mathrm{CCF}_{3}+\mathrm{HF}$ Übergangszustand $\mathrm{A}, \mathrm{B}$ und $\mathrm{C} / \mathrm{cm}^{-1}=0.06,0.04$ und 0.03; geschätzt aus der Geometrie, die auf dem MP2/6-31G(d) Level erhalten wird, aus [47]. 
$\mathrm{CF}_{3} \mathrm{CFHCF} \longrightarrow \mathrm{CF}_{3} \mathrm{H}+\mathrm{CF}_{3} \mathrm{CF}$ : Übergangszustand $\mathrm{A}, \mathrm{B}$ und $\mathrm{C} / \mathrm{cm}^{-1}=0.07,0.03$ und 0.02; geschätzt aus der Geometrie, die auf dem MP2/6-31G(d) Level erhalten wird, aus [4].

$\mathrm{H}+\mathrm{CF}_{3} \mathrm{CF}_{2} \mathrm{H} \longrightarrow \mathrm{H}_{2}+\mathrm{C}_{2} \mathrm{~F}_{5}$ : Übergangszustand $\mathrm{A}, \mathrm{B}$ und $\mathrm{C} / \mathrm{cm}^{-1}=0.115,0.076$ und 0.065; aus B3LYP/CBSB7 Berechnungen (aus CBS-QB3 Modell).

$\mathrm{CF}_{3}+\mathrm{CF}_{3} \mathrm{CF}_{2} \mathrm{H} \longrightarrow \mathrm{CF}_{3} \mathrm{H}+\mathrm{C}_{2} \mathrm{~F}_{5}$ : Übergangszustand $\mathrm{A}, \mathrm{B}$ und $\mathrm{C} / \mathrm{cm}^{-1}=0.051$, 0.021 und 0.020; aus B3LYP/CBSB7 Berechnungen (aus CBS-QB3 Modell). 



\section{Literaturverzeichnis}

[1] P. J. DiNenno, The SFPE Handbook of Fire Protection Engineering, (Hrsg.: P. J. DiNenno), Nat. Fire Protection Assn., 2002.

[2] H. R. G, J. C. Mackie, A. R. Masri, Combust. Flame 1998, 233, 554.

[3] H. R. G, J. C. Mackie, A. R. Masri, J. Phys. Chem. A 1999, 103, 54.

[4] B. A. Williams, D. M. L'Esperance, J. Fleming, Combust. Flame 2000, 120, 160.

[5] G. Copeland, E. P. F. Lee, J. M. Dyke, W. K. Chow, D. K. W. Mok, F. T. Chau, J. Phys. Chem. A 2010, 144, 3540.

[6] V. I. Babushok, G. T. Linteris, O. C. Meier, Combust. Flame 2012, 159, 3569.

[7] H. Hulpke, H. A. Koch, M. Adinolfi, ROEMPP-Lexikon Umwelt, 2. auflage Aufl., Georg Thieme Verlag, 2000.

[8] G. W. Gribble, Chemosphere 2003, 52, 289.

[9] E. Tschuikow-Roux, G. E. Millward, W. J. Quiring, J. Phys. Chem. 1971, 75, 3493.

[10] G. E. Millward, R. Hartig, E. Tschuikow-Roux, Chem. Commun. 1971, 456.

[11] K. Takahashi, A. Harada, S. Horigome, T. Inomata, Combust. Sci. Technol. 2007, 179, 1417.

[12] G. Copeland, E. P. F. Lee, J. M. Dyke, W. K. Chow, D. K. W. Mok, F. T. Chau, J. Phys. Chem. A 2010, 114, 1816.

[13] C. J. Cobos, A. E. Croce, K. Luther, L. Soelter, E. Tellbach, J. Troe, Experimental and Modeling Study of the Reaction C2F4 (+M) -> CF2 + CF2 $(+\mathrm{M})$, submitted 2013.

[14] (Hrsg.: A. Lifshitz), Shock Waves in Chemistry, (Hrsg.: A. Lifshitz), Marcel Dekker, Inc., New York, 1981.

[15] E. F. Greene, J. Toennies, Chemical Reactions in Shock Waves, E. Arnold, London, 1964.

[16] A. G. Gaydon, I. R. Hurle, The Shock Tube in High Temperature Chemical Physics, Chapman and Hall, London, 1963. 
[17] R. Courant, K. O. Friedrich, Supersonic Flow and Shock Waves, Interscience, New York, 1948.

[18] M. W. Chase, NIST-JANAF Thermochemical Tables, 9 Aufl., Bd. J. Phys. Chem. Ref. Data Monograph, (Hrsg.: M. W. C. ed.), Am. Inst. of Physics, Woodbury, New York, 1998.

[19] C. J. Cobos, A. E. Croce, K. Luther, J. Troe, Z. Phys. Chem. 2011, 225, 1019.

[20] C. J. Cobos, A. E. Croce, K. Luther, J. Troe, J. Phys. Chem. A 2010, 114, 47484754.

[21] F. Battin-Leclerc, A. P. Smith, G. D. Hayman, T. P. Murrells, Journal of the Chemical Society, Faraday Transactions 1996, 92(18), 3305, DOI 10.1039/ft9969203305.

[22] A. P. Modica, J. E. LaGraff, The Journal of Chemical Physics 1966, 45, 4729-4733.

[23] A. P. Modica, J. E. LaGraff, The Journal of Chemical Physics 1965, 43(9), 33833392.

[24] A. P. Modica, The Journal of Physical Chemistry 1968, 72, 4594-4598.

[25] K. P. Schug, H. G. Wagner, Ber. Bunsenges. Phys. Chem. 1978, 82, 719.

[26] G. A. Carlson, J. Phys. Chem. 1971, 75, 1625.

[27] D. C. Astholz, L. Brouwer, J. Troe, Ber. Bunsenges. Phys. Chem. 1981, 85, 559.

[28] C. J. Cobos, A. E. Croce, K. Luther, J. Troe, J. Phys. Chem. A 2010, 114, 4755-4791.

[29] V. Aviyente, Y. Inel, Can. J. Chem. 1990, 68, 1332.

[30] A. D. Boese, J. M. L. Martin, J. Chem. Phys. 2004, 121, 2405.

[31] Y. Zhao, D. G. Truhlar, Theor. Chem. Account 2008, 120, 215.

[32] J. A. Montgomery, M. J. Frisch, J. W. Ochterski, G. A. Petersson, J. Chem. Phys. $1999,110,2822$.

[33] A. G. Baboul, L. A. Curtiss, P. C. Redfern, K. Raghavachari, J. Chem. Phys. 1999, 110,7650 .

[34] L. A. Curtiss, P. C. Redfern, K. Raghavachari, J. Chem. Phys. 2007, 126, 084108.

[35] M. J. Frisch, G. W. Trucks, H. B. Schlegel, G. E. Scuseria, M. A. Robb, J. R. Cheeseman, G. Scalmani, V. Barone, B. Mennucci, G. A. Petersson, H. Nakatsuji, M. Caricato, X. Li, H. P. Hratchian, A. F. Izmaylov, J. Bloino, G. Zheng, J. L. Sonnenberg, M. Hada, M. Ehara, K. Toyota, R. Fukuda, J. Hasegawa, M. Ishida, T. Nakajima, Y. Honda, O. Kitao, H. Nakai, T. Vreven, J. A. Montgomery, Jr., J. E. Peralta, F. Ogliaro, M. Bearpark, J. J. Heyd, E. Brothers, K. N. Kudin, V. N. 
Staroverov, R. Kobayashi, J. Normand, K. Raghavachari, A. Rendell, J. C. Burant, S. S. Iyengar, J. Tomasi, M. Cossi, N. Rega, J. M. Millam, M. Klene, J. E. Knox, J. B. Cross, V. Bakken, C. Adamo, J. Jaramillo, R. Gomperts, R. E. Stratmann, O. Yazyev, A. J. Austin, R. Cammi, C. Pomelli, J. W. Ochterski, R. L. Martin, K. Morokuma, V. G. Zakrzewski, G. A. Voth, P. Salvador, J. J. Dannenberg, S. Dapprich, A. D. Daniels, . Farkas, J. B. Foresman, J. V. Ortiz, J. Cioslowski, D. J. Fox, Gaussian 09 Revision A.02-SMP, gaussian Inc. Wallingford CT 2009.

[36] E. Goos, A. Burcat, B. Ruscic, Extended Third Millenium Ideal Gas and Condensed Phase Thermochemical Tables Data Base for combustion with Updates from Active Thermochemical Tables, 2013.

[37] S. Kato, Y. Makide, T. Tominage, K. Takeuchi, J. Phys. Chem. 1987, 91, 4278.

[38] J. Troe, J. Phys. Chem. 1979, 83, 114.

[39] J. Troe, Ber. Bunsenges. Phys. Chem. 1983, 87, 161.

[40] R. G. Gilbert, K. Luther, J. Troe, Ber. Bunsenges. Phys. Chem. 1983, 87, 169.

[41] P. A. Hackett, C. Willis, M. Drowin, E. Weinberg, J. Phys. Chem. 1980, 84, 1873.

[42] S. Kato, Y. Makide, K. Takenchi, T. Tominaga, J. Phys. Chem. 1984, 88, 3977.

[43] T. Just, J. Troe, J. Phys. Chem. 1980, 84, 3068.

[44] R. D. Giles, L. M. Quick, E. Whittle, Trans. Faraday Soc. 1967, 63, 662.

[45] T. J. Wallington, M. D. Hurley, J. Shi, M. M. Maricq, J. Sehested, O. J. Nielsen, T. Ellermann, Int. J. Chem. Kinet. 1993, 25, 651.

[46] X. L. Zhao, Y. M. Ji, Z. S. Li, J. Y. Liu, J. Mol. Struc. 2007, 808, 17.

[47] S. D. Peterson, J. S. Fransicso, J. Phys. Chem. A 2002, 106, 3106.

[48] G. Copeland, E. P. F. Lee, J. M. Dyke, W. K. Chow, D. K. W. Mok, F. T. Chau, J. Phys. Chem. A 2010, 114, 2010. 



\section{Abbildungsverzeichnis}

2.1 Aufbau des Stoßwellenexperiments . . . . . . . . . . 12

2.2 Aufbau der Vormischapparatur . . . . . . . . . . . . . 13

2.3 Druckaufnehmersignal und Komparator-Ausgangssignal . . . . 15

3.1 Bestimmung des Absorptionsmaximums . . . . . . . . . . 19

3.2 Absorptionskoeffizient von $\mathrm{CF}_{2} \ldots \ldots \ldots \ldots \ldots \ldots$

3.3 Zerfall von Pentafluorethan bei $1269 \mathrm{~K} \ldots \ldots \ldots \ldots \ldots \ldots$

3.4 Zerfall von Pentafluorethan bei $1596 \mathrm{~K} \ldots \ldots \ldots \ldots \ldots$. . . . . 22

3.5 Zerfall von Pentafluorethan bei $2049 \mathrm{~K} \ldots \ldots \ldots \ldots \ldots \ldots$

3.6 Zerfall von Pentafluorethan bei $2017 \mathrm{~K} \ldots \ldots \ldots$. . . . . . 23

3.7 Absorptionskoeffizienten von Pentafluorethan und Heptafluorpropan 24

3.8 Zerfall von Pentafluorethan bei $2065 \mathrm{~K}$, kein Zerfall 1. Ordnung . 25

3.9 Bestimmung der Geschwindigkeitskonstanten aus der Anfangssteigung .................. 26

3.10 Arrhenius-Auftragung der Geschwindigkeitskonstante von $\mathrm{C}_{2} \mathrm{HF}_{5}$

3.11 Arrhenius-Auftragung der Geschwindigkeitskonstante von $\mathrm{C}_{3} \mathrm{HF}_{7}$

4.1 Vergleich der Messungen bei unterschiedlichen Konzentrationen 30

4.2 Vergleich der Messungen bei unterschiedlichen Konzentrationen 30

4.3 Arrheniusauftragung der Geschwindigkeitskonstanten von Pentafluo-

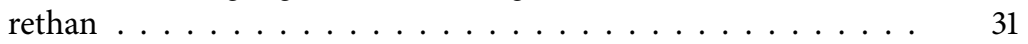

4.4 Energieprofil von Pentafluorethan . . . . . . . . . . . . 33

4.5 Arrheniusauftragung der effektiven Geschwindigkeitskonstanten der Pyrolyse von 2H-Heptafluorpropan . . . . . . . . . . 41

4.6 Vergleich von zwei Heptafluorpropan Einzelmessungen bei unterschiedlichen Konzentrationen . . . . . . . . . . . . . 42

4.7 Vergleich von zwei Heptafluorpropan Einzelmessungen bei unterschiedlichen Konzentrationen . . . . . . . . . . . . . 



\section{Tabellenverzeichnis}

2.1 Verwendete Chemikalien . . . . . . . . . . . . 15

3.1 Absorptionskoeffizienten . . . . . . . . . . . 19

4.1 Reaktionsenthalpien des Pentafluorethan . . . . . . . . . . 34

4.2 Barrierenhöhen des Pentafluorethan . . . . . . . . . . 34

4.3 Reaktionsenthalpien des 2H-Heptafluorpropan . . . . . . . 44

4.4 Barrierenhöhen des $2 \mathrm{H}$-Heptafluorpropan . . . . . . . . 44 
